TRANSACTIONS OF THE

AMERICAN MATHEMATICAL SOCIETY

Volume 281, Number I, January 1984

\title{
SEMIDIRECT PRODUCTS AND REDUCTION IN MECHANICS
}

BY

\author{
JERROLD E. MARSDEN ${ }^{1}$, TUDOR RATIU ${ }^{2}$ AND ALAN WEINSTEIN ${ }^{1}$
}

\begin{abstract}
This paper shows how to reduce a Hamiltonian system on the cotangent bundle of a Lie group to a Hamiltonian system in the dual of the Lie algebra of a semidirect product. The procedure simplifies, unifies, and extends work of Greene, Guillemin, Holm, Holmes, Kupershmidt, Marsden, Morrison, Ratiu, Sternberg and others. The heavy top, compressible fluids, magnetohydrodynamics, elasticity, the Maxwell-Vlasov equations and multifluid plasmas are presented as examples. Starting with Lagrangian variables, our method explains in a direct way why semidirect products occur so frequently in examples. It also provides a framework for the systematic introduction of Clebsch, or canonical, variables.
\end{abstract}

1. Introduction. This paper is a continuation of Marsden and Weinstein [1982a, 1982b], in which the methods of reduction were applied to the study of plasmas and incompressible fluids. Here we treat systems which are associated to semidirect products, including the heavy top, compressible fluids, magnetohydrodynamics and elasticity.

To discuss the results we need some notation.

$G$ is a Lie group.

$g$ is the Lie algebra of $G$.

$\rho$ is a left representation of $G$ on a vector space $V$.

$\rho_{*}$ is the associated left representation of $G$ on $V^{*}$ given by

$$
\rho_{*}(g)=\left[\rho\left(g^{-1}\right)\right] *
$$

where [ $]^{*}$ denotes the dual linear transformation. The right representation of $G$ on $V^{*}$ is given by

$$
\rho^{*}(g)=[\rho(g)]^{*} .
$$

$\rho^{\prime}: \mathfrak{g} \rightarrow \operatorname{End}(V)$ is the induced Lie algebra representation.

$S=G \times{ }_{\rho} V$ is the semidirect product and $\mathfrak{s}$ is its Lie algebra.

$G_{a}$ is the stabilizer of $a \in V^{*}$ under $\rho^{*}$.

Semidirect products were shown to be relevant for the heavy top in Vinogradov and Kupershmidt [1977], Guillemin and Sternberg [1980] and Ratiu [1980]. In Ratiu $[1980,1981,1982]$ it was shown that reducing $T^{*} G$ by the left action of $G_{a}$, in the

Received by the editors September 17, 1982.

1980 Mathematics Subject Classification. Primary 58F05, 58D30, 58G16, 70H05, 73C50, 76A02; Secondary 22E70, 35L 70, 58B25, 58D05.

'Research partially supported by NSF grants MCS 81-07086, MCS 80-23356, the Miller Institute and DOE contract DE-AT03-82ER12097.

${ }^{2}$ Research partially supported by NSF grant MCS $81-01642$.

(C)1984 American Mathematical Society $0002-9947 / 84 \$ 1.00+\$ .25$ per page 
sense of Marsden and Weinstein [1974], leads to a coadjoint orbit for $S$. The proof was simplified in Holmes and Marsden [1983], using some ideas from Guillemin and Sternberg [1980]. Using results from Kazhdan, Kostant and Sternberg [1978], Guillemin and Sternberg [1982] made further improvements.

Here we extend and simplify these results. In the process, we unify the RatiuGuillemin-Sternberg results with a map introduced by Kupershmidt [1982]. We show that the right and left actions of $S$ on $T^{*} S$ yield a dual pair, and that their momentum maps induce symplectic diffeomorphisms of the reduced spaces with coadjoint orbits. This approach also leads to canonical (or Clebsch) variables in a simple way; see Marsden and Weinstein [1982b] for related results.

The relation between coadjoint orbits and isotropy groups may be seen as a classical analogue of the Mackey-Wigner "little group" induction procedure. (A little group is just an isotropy group.) This fact is also mentioned by Guillemin and Sternberg [1980], referring to Rawnsley [1975] for an explicit link via geometric quantization.

We recall some additional notation from Marsden and Weinstein [1982a, 1982b]. For a Lie group $G$, the Lie algebra $g$ may be identified with the left invariant vector fields on $G$ with the commutator bracket $[X, Y]=X Y-Y X$. We let $g_{-}^{*}$ denote the manifold $\mathrm{g}^{*}$ with the Poisson bracket structure $C^{\infty}\left(\mathfrak{g}^{*}\right)$ given by

$$
\{F, G\}_{-}(\mu)=-\langle\mu,[\delta F / \delta \mu, \delta G / \delta \mu]\rangle .
$$

The "functional derivative" $\delta F / \delta \mu \in \mathfrak{g}$ is the derivative $D F(\mu)$ regarded as an element of $\mathfrak{g}$ rather than $\mathfrak{g}^{* *}$; i.e.

$$
D F(\mu) \cdot \nu=\langle\nu, \delta F / \delta \mu\rangle
$$

where $\langle$,$\rangle denotes the pairing between \mathrm{g}^{*}$ and $\mathrm{g}$. We call $\{F, G\}_{-}$the Lie-Poisson bracket. The Lie-Poisson manifold $\mathfrak{g}_{-}^{*}$ is identifiable with the reduced Poisson manifold $T^{*} G / G$ for the left action of $G$. We shall make this identification explicit in $\S 2$. Likewise, the reduced manifold for the right action ${ }^{3}$ of $G$ on $T^{*} G$ is $g_{+}^{*}$ with the bracket

$$
\{F, G\}_{+}(\mu)=\langle\mu,[\delta F / \delta \mu, \delta G / \delta \mu]\rangle .
$$

If $\tilde{H}$ is the left invariant Hamiltonian on $T^{*} G$, it naturally induces a reduced Hamiltonian $H$ of $\mathfrak{g}_{-}^{*}$ and a reduced Hamiltonian vector field $X_{H}$ on $\mathrm{g}_{-}^{*}$ given by

$$
X_{H}(\mu)=\operatorname{ad}(\delta H / \delta \mu) * \mu
$$

where $\operatorname{ad}(\xi) \eta=[\xi, \eta]$ and $\operatorname{ad}(\xi)^{*}$ is the adjoint of $\operatorname{ad}(\xi): \mathfrak{g} \rightarrow \mathfrak{g}$. The evolution equation $\dot{\mu}=X_{H}(\mu)$ for $X_{H}$ is equivalent to that determined by the Lie-Poisson equations

$$
\dot{F}=\{F, H\}_{-} .
$$

\footnotetext{
${ }^{3}$ One could make things more symmetric by using the left and right Lie algebras, but we are pretty much stuck with the left Lie algebra by convention.
} 
To see this, we note that for any smooth $F: \mathrm{g}^{*} \rightarrow \mathbf{R}$ the left-hand side of (1.4) equals $\dot{F}(\mu)=D F(\mu) \cdot \dot{\mu}=\langle\dot{\mu}, \delta F / \delta \mu\rangle$ whereas the right-hand side is $\{F, H\}_{-}(\mu)=$ $-\langle\mu,[\delta F / \delta \mu, \delta H / \delta \mu]\rangle=\left\langle\operatorname{ad}(\delta H / \delta \mu)^{*} \mu, \delta F / \delta \mu\right\rangle$; in other words, since $\dot{\mu}=X_{H}(\mu)$, (1.3) is equivalent to (1.4). In practice, to get the equations of motion one uses (1.4) on appropriately chosen functions $F$, since this usually entails less computation than the determination of the adjoint in (1.3); this remark is especially handy in infinite dimensions.

Each trajectory of $X_{H}$ stays in the coadjoint orbit of its initial condition. This is often viewed as a "constraint". For example, for compressible fluids discussed in $§ 5$, this is the "Lin constraint", cf. Bretherton [1970].

Now suppose that $H$ is a Hamiltonian on $T^{*} G$, depending parametrically on $a \in V^{*}$, which is left $G_{a}$-invariant for each $a$. We shall prove that $H$ induces a Lie-Poisson system on $\mathfrak{3}^{*}$. If $H$ is right invariant, then the induced system lives on $\mathfrak{S}_{+}^{*}$ instead. We wish to emphasize the fact that the constructions used to obtain this result are all natural.

It is clear from Vinogradov and Kupershmidt [1977], Guillemin and Sternberg [1980] and Ratiu [1980] that the equations for a heavy top are Lie-Poisson equations for the Euclidean group $E(3)=\mathrm{SO}(3) \times \mathbf{R}^{3}$. Guillemin and Sternberg [1980] approached the problem from the point of view of collective motion; here we approach it, as in Ratiu [1980], by reduction. (See Holmes and Marsden [1983] for a "bare hands" verification in this case and an application to chaotic motion.)

By 1980 it was known that the equations for compressible flow on $\mathbf{R}^{3}$ were formally Lie-Poisson equations for the semidirect product $\mathscr{D} \times \mathcal{F}$, where $\mathscr{D}$ is the diffeomorphism group of $\mathbf{R}^{3}$, and $\mathscr{F}$ is the space of functions on $\mathbf{R}^{3}$. This fact is hinted at in Guillemin and Sternberg [1980] and is easily verified. Almost simultaneously, the bracket for this system and the MHD system was published by Morrison and Greene [1980] and Dzyaloshinskii and Volovick [1980]. For MHD and elasticity, the explicit connection with semidirect product Lie-Poisson structures was made by Holm and Kupershmidt [1982]. Their approach is quite different from ours; they begin with a variational principle using the constraints and Clebsch variables, as in Seliger and Whitham [1968], and derive a reduced bracket from a canonical bracket in the Clebsch variables. Finally, they simply observe that the bracket is a Lie-Poisson bracket for an appropriate semidirect product. Our approach is the reverse: the semidirect product structure is derived directly in terms of the physical variables and reduction; constraints appear in terms of coadjoint orbits, and Clebsch variables occur as momentum maps.

the semidirect product structure is derived directly in terms of the physical variables and reduction; constraints appear in terms of coadjoint orbits, and Clebsch variables occur as momentum maps.

In the last section we show that there is a semidirect product structure in the momentum representation of the Poisson brackets for the Maxwell-Vlasov and multifluid plasma equations. This structure arises by a rather different construction than that sketched above; the momentum representation is closely related to a construction of Sternberg [1977], whereas the usual velocity representation is closer to the construction of Weinstein [1978]. 
The main body of the paper will use some further facts and notation. Proofs may be found in Abraham and Marsden [1978], Guillemin and Sternberg [1980, 1982] and Marsden and Weinstein [1982b].

If $\varphi: M \rightarrow N$ is a diffeomorphism, $\varphi_{*}: T^{*} M \rightarrow T^{*} N$ denotes its canonical lift, defined on the fiber $T_{x}^{*} M$ over $x \in M$ by

$$
\left(\varphi_{*}\right)_{x}=\left[\left(T_{\varphi(x)} \varphi\right)^{-1}\right] *
$$

A lower star is used since $\varphi_{*}$ pushes covectors forward. If $G$ acts from the left (resp. right) on $M$, then by lifting we get a left (resp. right) symplectic action of $G$ on $T^{*} M$. This action has a momentum map $J: T^{*} M \rightarrow \mathrm{g}^{*}$, given by

$$
\left\langle J\left(\alpha_{x}\right), \xi\right\rangle=\left\langle\alpha_{x}, \xi_{M}(x)\right\rangle
$$

where $\xi_{M}$ is the infinitesimal generator for $\xi \in g$ and the action of $G$ on $M$. The map

$$
J: T^{*} M \rightarrow \mathrm{g}_{ \pm}^{*}
$$

is a Poisson map (i.e. preserves Poisson brackets), where " +" is used for a left action and " - " for a right action.

If $(P, \omega)$ is a symplectic manifold and $G$ acts by canonical transformations on the left (resp. right), an Ad*-equivariant momentum map is a Poisson map $J: P \rightarrow \mathrm{g}_{ \pm}^{*}$ such that $X_{\hat{J}(\xi)}=\xi_{P}$ for $\xi \in g$ where $\hat{J}(\xi)(p)=\langle J(p), \xi\rangle$ and $\xi_{P}$ is the infinitesimal generator defined by $\xi$ for the action of $G$ on $P$. The reduced space $P_{\mu}$ is $J^{-1}(\mu) / G_{\mu}$, where $\mu \in \mathrm{g}^{*}$ is a regular value of $J$, and $G_{\mu}$ is assumed to act freely and properly. (Without these assumptions, $P_{\mu}$ can have singularities.) If $\theta_{\mu}$ is the coadjoint orbit of $\mu$,

$$
P_{\mu} \approx J^{-1}\left(\vartheta_{\mu}\right) / G
$$

and these are the symplectic leaves in the reduced Poisson manifold $P / G$.

General reductions can be replaced by reductions at zero by changing $P$. Indeed $P_{\mu}$ is symplectically diffeomorphic to $\left(P \ominus \theta_{\mu}^{+}\right)_{0}$ and to $\left(P \oplus \theta_{\mu}^{-}\right)_{0}$. For right actions, ${ }_{\mu} P:=G_{\mu} \backslash J^{-1}(\mu) \approx{ }_{0}\left(P \ominus \vartheta_{\mu}^{-}\right) \approx{ }_{0}\left(P \oplus \theta_{\mu}^{+}\right)$.

2. Left and right reductions of cotangent bundles of Lie groups. This section summarizes the reduction of $T^{*} G$ by the right and left actions of $G$.

If $L_{g}$ and $R_{g}$ denote left and right translation by $g$ in $G$, these actions can be lifted to left and right actions on $T^{*} G$ as follows. Define

$$
L: G \times T^{*} G \rightarrow T^{*} G, \quad L\left(g, \alpha_{h}\right)=\left(T_{g h} L_{g^{-1}}\right)^{*} \alpha_{h}
$$

and

$$
R: T^{*} G \times G \rightarrow T^{*} G, \quad R\left(\alpha_{h}, g\right)=\left(T_{h g} R_{g^{-1}}\right)^{*} \alpha_{h} .
$$

These two commuting actions have the (Ad*-equivariant) momentum maps

$$
J_{L}: T^{*} G \rightarrow \mathrm{g}^{*}, \quad J_{L}\left(\alpha_{g}\right)=\left(T_{e} R_{g}\right)^{*}\left(\alpha_{g}\right)
$$

and

$$
J_{R}: T^{*} G \rightarrow \mathrm{g}^{*}, \quad J_{R}\left(\alpha_{g}\right)=\left(T_{e} L_{g}\right) *\left(\alpha_{g}\right) .
$$


Thus

$$
J_{L}: T^{*} G \rightarrow \mathrm{g}_{+}^{*} \text { and } J_{R}: T^{*} G \rightarrow \mathrm{g}_{-}^{*}
$$

are Poisson maps. Moreover, $J_{L}$ is $R$-invariant and $J_{R}$ is $L$-invariant so that these maps induce Poisson manifold diffeomorphisms on the corresponding quotient spaces

$$
\bar{J}_{L}: R \backslash T^{*} G \rightarrow \mathrm{g}_{+}^{*} \quad \text { and } \quad \bar{J}_{R}: T^{*} G / L \rightarrow \mathrm{g}_{-}^{*}
$$

with inverses $\bar{J}_{L}^{-1}(\mu)=[\mu]_{R}, \bar{J}_{R}^{-1}(\mu)=[\mu]_{L} \cdot\left([\mu]_{R}\right.$ and $[\mu]_{L}$ denote equivalence classes in $R \backslash T^{*} G, T^{*} G / L$.) Denote the canonical projections by $\pi_{L}: T^{*} G \rightarrow T^{*} G / L$ and $\pi_{R}: T^{*} G \rightarrow R \backslash T^{*} G$.

The symplectic leaves of $g^{*}$ are the coadjoint orbits. In $g_{ \pm}^{*}$ the orbit $\theta_{\mu}^{ \pm}$through $\mu \in \mathrm{g}^{*}$ has the Lie-Berezin-Kirillov-Arnold-Kostant-Souriau symplectic form

$$
\left((\operatorname{ad} \xi)^{*} \nu,(\operatorname{ad} \eta)^{*} \nu\right)= \pm \nu \cdot[\xi, \eta]
$$

where $\nu \in \mathcal{O}_{\mu}^{ \pm}$and $\xi, \eta \in g$. Hence the symplectic leaves of $T^{*} G / L$ are of the form $\bar{J}_{R}^{-1}\left(\theta_{\mu}^{-}\right)$, and those of $R \backslash T^{*} G$ are of the form $\bar{J}_{L}^{-1}\left(\theta_{\mu}^{+}\right)$. But it is easily seen that

$$
\bar{J}_{R}^{-1}\left(\theta_{\mu}^{-}\right)=\pi_{L} J_{L}^{-1}\left(\theta_{\mu}^{+}\right), \quad \bar{J}_{R}^{-1}\left(\theta_{\mu}^{+}\right)=\pi_{R} J_{R}^{-1}\left(\theta_{\mu}^{-}\right)
$$

and, hence, the symplectic leaves in $T^{*} G / L$ and $R \backslash T^{*} G$ are the reduced manifolds $\pi_{L} J_{L}^{-1}\left(\theta_{\mu}^{+}\right)$and $\pi_{R} J_{R}^{-1}\left(\theta_{\mu}^{-}\right)$, where $\theta_{\mu}^{ \pm}$has the " + " or " $-"$ Lie-Poisson symplectic form. We summarize these results in the following theorem.

2.1. TheOREM. The Ad*-equivariant momentum maps $J_{L}$ and $J_{R}$ for the actions $L$ and $R$ of $G$ on $T^{*} G$ induce Poisson manifold diffeomorphisms

$$
\bar{J}_{L}: R \backslash T^{*} G \rightarrow \mathrm{g}_{+}^{*} \text { and } \bar{J}_{R}: T^{*} G / L \rightarrow \mathrm{g}_{-}^{*}
$$

where $\mathrm{g}_{ \pm}^{*}$ is endowed with the " \pm " Lie-Poisson bracket. The symplectic leaves in the quotient spaces $T^{*} G / L$ and $R \backslash T^{*} G$ are the reduced manifolds $J_{L}^{-1}\left(\theta_{\mu}^{+}\right) / L$ and $R \backslash J_{R}^{-1}\left(\theta_{\mu}^{-}\right)$where $\mu \in \mathrm{g}^{*}$ and $\theta_{\mu}^{ \pm}$is the coadjoint orbit of $G$ in $\mathrm{g}^{*}$ through $\mu$ with the " \pm " Lie-Poisson symplectic form.

In particular, note that the actions $L$ and $R$ form a dual pair i.e. the reduced spaces for one action are coadjoint orbits for the other and we get the diagram:

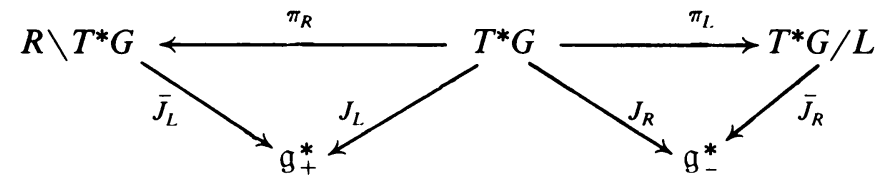

Now, assume that $H: T^{*} G \rightarrow \mathbf{R}$ is a Hamiltonian invariant under the lifted action $L$ on $T^{*} G$ of the left translation on $G$. Then $H$ induces a smooth mapping $H_{L}$ : $T^{*} G / L \rightarrow \mathbf{R}$, and hence the function $H_{-}=H_{L} \circ \bar{J}_{R}^{-1}: g_{-}^{*} \rightarrow \mathbf{R}$ defines Lie-Poisson equations on $\mathrm{g}_{-}^{*}$. Since Lie-Poisson equations have trajectories which remain in the coadjoint orbits of their initial conditions, $H_{L}$ when restricted to the reduced manifolds $J_{L}^{-1}\left(\theta_{\mu}^{+}\right) / L$, where $\mu \in \mathrm{g}^{*}$, defines Hamiltonian systems on these manifolds. Moreover, if $F_{t}$ denotes the flow of $X_{H}$, and $G_{t}^{-}$is the flow of the Lie-Poisson equation defined by $H_{-}$, we have $G_{t}^{-} \circ \bar{J}_{R} \circ \pi_{L}=\bar{J}_{R} \circ \pi_{L} \circ F_{t}$. We summarize these results in the following theorem. 
2.2. Theorem. A left invariant Hamiltonian $H: T^{*} G \rightarrow \mathbf{R}$ canonically induces Lie-Poisson equations on $\mathrm{g}_{-}^{*}$ defined by the Hamiltonian function $H_{-}=H_{L} \circ \bar{J}_{R}^{-1}$ where $H_{L} \circ \pi_{L}=H$. If $F_{t}$ denotes the flow of $X_{H}$ and $G_{t}^{-}$is the flow of the Lie-Poisson equations for $H_{-}$on $\mathrm{g}_{-}^{*}$, then $G_{t}^{-} \circ \bar{J}_{R} \circ \pi_{L}=\bar{J}_{R} \circ \pi_{L} \circ F_{t}$. The Hamiltonian $H_{L} \mid\left(J_{L}^{-1}\left(\theta_{\mu}^{+}\right) / L\right)$, where $\mu \in \mathrm{g}^{*}$, induces a Hamiltonian system on the reduced manifold $J^{-1}\left(\theta_{\mu}^{+}\right) / L$. The same result holds if "left" and "right", and " - " and " +" are interchanged.

3. Semidirect products. We now apply the results of the previous section to the case of semidirect products. Recall from $\S 1$ that $\rho: G \rightarrow \operatorname{Aut}(V)$ denotes a (left) Lie group representation of $G$ in the vector space $V$, and $\rho^{\prime}: \mathfrak{g} \rightarrow \operatorname{End}(V)$ is the induced Lie algebra representation. Denote by $S$ the semidirect product group of $G$ with $V$ by $\rho$ with multiplication

$$
\left(g_{1}, u_{1}\right)\left(g_{2}, u_{2}\right)=\left(g_{1} g_{2}, u_{1}+\rho\left(g_{1}\right) u_{2}\right) .
$$

Let $\mathfrak{s}=\mathfrak{g} \times_{\rho^{\prime}} V$ be the Lie algebra of $S$. The Lie bracket on $\mathfrak{g}$ is given by

$$
\left[\left(\xi_{1}, v_{1}\right),\left(\xi_{2}, v_{2}\right)\right]=\left(\left[\xi_{1}, \xi_{2}\right], \rho^{\prime}\left(\xi_{1}\right) v_{2}-\rho^{\prime}\left(\xi_{2}\right) v_{1}\right) .
$$

The adjoint and coadjoint actions of $S$ on $\mathfrak{s}$ and $\mathfrak{s}^{*}$ are given by

$$
\operatorname{Ad}_{(g, u)}(\xi, v)=\left(\operatorname{Ad}_{g} \xi, \rho(g) v-\rho^{\prime}\left(\operatorname{Ad}_{g} \xi\right) u\right)
$$

and

$$
\left[\operatorname{Ad}_{(g, u)^{-1}}\right]^{*}(\nu, a) \equiv \operatorname{Ad}_{(g, u)^{-1}}^{*}(\nu, a)=\left(\operatorname{Ad}_{g^{-1}}^{*} \nu+\left(\rho_{u}^{\prime}\right)^{*}\left(\rho_{*}(g)\right) a, \rho_{*}(g) a\right),
$$

where $g \in G, u, v \in V, \nu \in \mathfrak{g}^{*}$, and $a \in V^{*} ; \rho_{u}^{\prime}: \mathfrak{g} \rightarrow V$ is given by $\rho_{u}^{\prime}(\xi)=\rho^{\prime}(\xi) u$ and $(g, u)^{-1}=\left(g^{-1},-\rho\left(g^{-1}\right) u\right)$. The “ \pm " Lie-Poisson bracket of $F, G: \mathfrak{g}^{*} \rightarrow \mathbf{R}$ is

$$
\{F, G\}_{ \pm}(\mu, a)= \pm\left\langle\mu,\left[\frac{\delta F}{\delta \mu}, \frac{\delta G}{\delta \mu}\right]\right\rangle \pm\left\langle a, \rho^{\prime}\left(\frac{\delta F}{\delta \mu}\right) \cdot \frac{\delta G}{\delta a}\right\rangle \mp\left\langle a, \rho^{\prime}\left(\frac{\delta G}{\delta \mu}\right) \cdot \frac{\delta F}{\delta a}\right\rangle
$$

where, as in $\S 1, \delta F / \delta \mu \in \mathfrak{g}$ and $\delta F / \delta a \in V$. Also from formula (1.3), we compute the Hamiltonian vector field of $H: \mathfrak{S}^{*} \rightarrow \mathbf{R}$ to be

$$
X_{H}(\mu, a)=\mp\left(\operatorname{ad}\left(\frac{\delta H}{\delta \mu}\right)^{*} \mu-\left(\rho_{\delta H / \delta a}^{\prime}\right)^{*} a, \rho^{\prime}\left(\frac{\delta H}{\delta \mu}\right)^{*} a\right)
$$

where $\rho_{\delta H / \delta a}^{\prime}: \mathrm{g} \rightarrow V$ is given by $\rho_{\delta H / \delta a}^{\prime}(\xi)=\rho^{\prime}(\xi) \cdot \delta H / \delta a$, and $\left(\rho_{\delta H / \delta a}^{\prime}\right)^{*}$ is its adjoint.

We shall explicitly compute the actions $L$ and $R$ of the previous section for the group $S$. Since

$$
L_{(g, u)}(h, v)=(g h, u+\rho(g) v)
$$

we have

$$
T_{(h, v)} L_{(g, u)}\left(v_{h}, v, w\right)=\left(T_{h} L_{g}\left(v_{h}\right), u+\rho(g) v, \rho(g) w\right)
$$

for $\left(v_{h}, v, w\right) \in T_{(h, v)}(G \times V)$. Thus

$$
T_{(g, u)(h, v)} L_{(g, u)^{-1}}\left(v_{g h}, u+\rho(g) v, w\right)=\left(T_{g h} L_{g^{-1}}\left(v_{g h}\right), v, \rho\left(g^{-1}\right) w\right)
$$




$$
\begin{aligned}
& \text { for }\left(v_{g h}, u+\rho(g) v, w\right) \in T_{(g, u)(h, v)}(G \times V) \text { and, hence, } \\
& \qquad \begin{aligned}
L\left((g, u),\left(\alpha_{h}, v, a\right)\right) & =\left(T_{(g, u)(h, v)} L_{(g, u)^{-1}}\right) *\left(\alpha_{h}, v, a\right) \\
& =\left(\left(T_{g h} L_{g^{-1}}\right)^{*} \alpha_{h}, u+\rho(g) v, \rho_{*}(g) a\right)
\end{aligned}
\end{aligned}
$$

for $\left(\alpha_{h}, v, a\right) \in T_{(h, v)}^{*}(G \times V)$. Since $R_{(g, u)}(h, v)=(h g, v+\rho(h) u)$, we have

$$
T_{(h, v)} R_{(g, u)}\left(V_{h}, v, w\right)=\left(T_{h} R_{g}\left(v_{h}\right), v+\rho(h) u, w+T_{h} \rho\left(v_{h}\right) \cdot u\right)
$$

for $\left(v_{h}, v, w\right) \in T_{(h, v)}(G \times V)$. Thus,

$$
\begin{aligned}
T_{(h, v)(g, u)} R_{(g, u)^{-1}} & \left(v_{h g}, v+\rho(h) u, w\right) \\
& =\left(T_{h g} R_{g^{-1}}\left(v_{h g}\right), v, w-T_{h g} \rho\left(v_{h g}\right) \cdot \rho\left(g^{-1}\right) u\right)
\end{aligned}
$$

for $\left(v_{h g}, v+\rho(g) u, w\right) \in T_{(h, v)(g, u)}(G \times V)$ and, hence,

$$
\begin{aligned}
R\left((g, u),\left(\alpha_{h}, v, a\right)\right) & =\left(T_{(h, v)(g, u)} R_{(g, u)^{-1}}\right) *\left(\alpha_{h}, v, a\right) \\
& =\left(\left(T_{h g} R_{g^{-1}}\right)^{*} \alpha_{h}-d f_{\rho\left(g^{-1}\right) u}^{a}(h g), v+\rho(h) u, a\right)
\end{aligned}
$$

where $f_{u}^{a}(g)$ is the "matrix element" $\langle a, \rho(g) u\rangle$. The last equality is obtained by applying the left-hand side to $\left(v_{h g}, v+\rho(g) u, w\right) \in T_{(h, v)(g, u)}(G \times V)$ and using the easily verifiable formula

$$
\left\langle a, T_{k} \rho\left(v_{k}\right) z\right\rangle=d f_{z}^{a}(k) v_{k}
$$

for $a \in V^{*}, k \in G, z \in V, v_{k} \in T_{k} G$.

The corresponding momentum mappings are

$$
\begin{gathered}
J_{L}: T^{*} S \rightarrow \mathfrak{S}_{+}^{*}, \\
J_{L}\left(\alpha_{g}, v, a\right)=\left(T_{(e, 0)} R_{(g, v)}\right)^{*}\left(\alpha_{g}, v, a\right)=\left(\left(T_{e} R_{g}\right) * \alpha_{g}+\left(\rho_{v}^{\prime}\right)^{*} a, a\right)
\end{gathered}
$$

and

$$
\begin{gathered}
J_{R}: T^{*} S \rightarrow \mathfrak{S}_{-}^{*}, \\
J_{R}\left(\alpha_{g}, v, a\right)=\left(T_{(e, o)} L_{(g, v)}\right) *\left(\alpha_{g}, v, a\right)=\left(\left(T_{e} L_{g}\right)^{*} \alpha_{g}, \rho^{*}(g) a\right) .
\end{gathered}
$$

The results of the previous section apply directly to this situation. Thus we get, for example,

3.1. Proposition. Let $J_{L}$ and $J_{R}$ be given by (3.3) and (3.4). Then:

(1) $J_{L}$ and $J_{R}$ are Poisson maps.

(2) $J_{L}\left(\right.$ resp. $\left.J_{R}\right)$ induces a Poisson diffeomorphism of $R \backslash T^{*} S\left(\right.$ resp. $\left.T^{*} S / L\right)$ with $\mathfrak{s}_{+}^{*}\left(\right.$ resp. $\left.\mathfrak{s}_{-}^{*}\right)$.

(3) The reduced spaces for the $R$ (resp. L) action are coadjoint orbits in $\mathfrak{S}_{+}^{*}$ (resp. $\left.\mathfrak{S}_{-}^{*}\right)$.

Now we shall relate our results to those in the literature. The following lemma will be useful. 
3.2. LemmA. Let $\alpha: G_{1} \rightarrow G_{2}$ be a Lie group homomorphism and $\phi:\left(P_{1}, \omega_{1}\right) \rightarrow$ $\left(P_{2}, \omega_{2}\right)$ a symplectic mapping. Assume that $G_{1}$ acts on $P_{1}, G_{2}$ acts on $P_{2}$, and $(\phi, \alpha)$ intertwines these actions, i.e.

$$
\phi\left(g \cdot p_{1}\right)=\alpha(g) \cdot \phi\left(p_{1}\right)
$$

where "." denotes the appropriate action. If $J_{2}: P_{2} \rightarrow \mathrm{g}_{2}^{*}$ is a momentum map for the $G_{2}$ action, then $J_{1}=\left(\alpha^{\prime}\right)^{*} \circ J_{2} \circ \phi$ is a momentum map for the $G_{1}$ action.

Proof. First of all, note that

$$
\hat{J}_{1}(\xi)=\hat{J}_{2}\left(\alpha^{\prime} \cdot \xi\right) \circ \phi
$$

and so, for $v_{p_{1}} \in T_{p_{1}} P_{1}$,

$$
d \hat{J}_{1}(\xi) \cdot v_{p_{1}}=d \hat{J}_{2}\left(\alpha^{\prime} \cdot \xi\right) \cdot T \phi\left(v_{p_{1}}\right)=\omega_{2}\left(\left(\alpha^{\prime} \cdot \xi\right)_{P_{2}}\left(\phi\left(p_{1}\right)\right), T \phi\left(v_{p_{1}}\right)\right) .
$$

Now $\phi\left(g \cdot p_{1}\right)=\alpha(g) \cdot \phi\left(p_{1}\right)$ gives $T \phi\left(\xi_{p_{1}}\left(p_{1}\right)\right)=\left(\alpha^{\prime} \cdot \xi\right)_{P_{2}}\left(\phi\left(p_{1}\right)\right)$ and so

$$
d \hat{J}_{1}(\xi) \cdot v_{p_{1}}=\omega_{2}\left(T \phi\left(\xi_{p_{1}}\left(p_{1}\right)\right), T \phi\left(v_{p_{1}}\right)\right)=\omega_{1}\left(\xi_{P_{1}}\left(p_{1}\right), v_{p_{1}}\right)
$$

since $\phi$ is symplectic. Thus $X_{\hat{J}_{1}(\xi)}=\xi_{P_{1}}$.

Finally, $J_{1}$ is a Poisson map because each of $\left(\alpha^{\prime}\right)^{*}, J_{2}$ and $\phi$ are.

Remarks. (1) Consider the isomorphism $\alpha: S \rightarrow S$ given by $\alpha(g, u)=(g,-u)$ and the left action $L_{\alpha(g, u)}$ of $S$ on $T^{*} S$. Its momentum map is (see Lemma 3.2)

$$
\left(\beta_{g}, u, a\right) \mapsto\left(\alpha^{\prime}\right)^{*} J_{L}\left(\beta_{g}, u, a\right)=\left(\left(T_{e} R_{g}\right)^{*} \beta_{g}+\left(\rho_{u}^{\prime}\right)^{*} a,-a\right) .
$$

The induced Poisson map of $V \times V^{*} \rightarrow \mathfrak{I}_{+}^{*}$, given by $(u, a) \mapsto\left(\left(\rho_{u}^{\prime}\right)^{*} a,-a\right)$, has been used by Kupershmidt [1982] in connection with Clebsch variables (see $\S 4$ below).

(2) Guillemin and Sternberg [1980] consider the following left action of $S$ on $T^{*} G$ for fixed $a \in V^{*}$ :

$$
\Gamma\left((g, u), \alpha_{h}\right)=\left(T_{g h} L_{g^{-1}}\right) * \alpha_{h}-d \phi_{u}^{a}(g h)
$$

where $\phi_{u}^{a}(g)=\left\langle a, \rho\left(g^{-1}\right) u\right\rangle$. In the present context this action is understood in the following way.

Given $G, V$ and the representation $\rho$, one forms the semidirect product $S$ and its Lie algebra $\mathfrak{G}$. However, there is another group $\tilde{S}$ with the same Lie algebra $\mathfrak{g}$; $S=G \times V$ as a manifold, but the composition law is

$$
\left(g_{1}, u_{1}\right) \circ\left(g_{2}, u_{2}\right)=\left(g_{1} g_{2}, u_{2}+\rho\left(g_{2}^{-1}\right) u_{1}\right)
$$

with identity element $(e, 0)$, and inverse $(g, u)^{-1}=\left(g^{-1},-\rho(g) u\right)$. In fact, $\beta: S \rightarrow \tilde{S}$ given by $\beta(g, u)=\left(g, \rho\left(g^{-1}\right) u\right)$ is a Lie group isomorphism whose induced Lie algebra isomorphism $\beta^{\prime}: \mathfrak{g} \rightarrow \mathfrak{F}$ is easily seen to be the identity ( $\mathfrak{g}$ is the Lie algebra of $\tilde{S}$ ). Thus one would expect to get different lifts to $T^{*} S$, but with the same momentum map. This is indeed the case as we shall see below.

If $\tilde{L}_{(g, u)}\left(\tilde{R}_{(g, u)}\right)$ denotes left (right) translation by $(g, u)$ in $\tilde{S}$, an easy computation shows that

$$
T_{(h, v)} \tilde{L}_{(g, u)}\left(v_{h}, v, w\right)=\left(T_{h} L_{g}\left(v_{h}\right), v+\rho\left(h^{-1}\right) u, w+T_{h}(\rho \circ \iota)\left(v_{h}\right) \cdot u\right)
$$


where $\iota: G \rightarrow G$ is the inversion map $g \mapsto g^{-1}$. Thus,

$$
\left(T_{(h, v)} \tilde{L}_{(g, u)}\right) *\left(\alpha_{g h}, v+\rho\left(h^{-1}\right) u, a\right)=\left(\left(T_{h} L_{g}\right)^{*} \alpha_{g h}+d \phi_{u}^{a}(h), v, a\right)
$$

for $\left(\alpha_{g h}, v+\rho\left(h^{-1}\right) u, a\right) \in T_{(g, u) \circ(h, v)}^{*} \tilde{S}$. Thus, the lift to $T^{*} \tilde{S}$ of left translation on $\tilde{S}$ is given by

$$
\begin{aligned}
\tilde{L}\left((g, u),\left(\alpha_{h}, v, a\right)\right) & =\left(T_{(g, u) \circ(h, v)} \tilde{L}_{(g, u)^{-1}}\right) *\left(\alpha_{h}, v, a\right) \\
& =\left(\left(T_{g h} L_{g^{-1}}\right)^{*} \alpha_{h}-d \phi_{\rho(g) u}^{a}(g h), v+\rho\left(h^{-1}\right) u, a\right)
\end{aligned}
$$

and has momentum map $\tilde{J}_{L}: T^{*} \tilde{S} \rightarrow \mathfrak{S}_{+}^{*}$ given by

$$
\tilde{J}_{L}\left(\alpha_{g}, v, a\right)=\left(T_{(e, 0)} \tilde{R}_{(g, v)}\right) *\left(\alpha_{g}, v, a\right)=\left(\left(T_{e} R_{g}\right)^{*} \alpha_{g}, \rho_{*}(g) a\right) .
$$

Now define the left action $\tilde{\Gamma}$ of $S$ on $T^{*} S$ by $\tilde{\Gamma}_{(g, u)}=\tilde{L}_{\beta(g, u)}$, i.e.

$$
\tilde{\Gamma}\left((g, u),\left(\alpha_{h}, v, a\right)\right)=\left(\left(T_{g h} L_{g^{-1}}\right)^{*} \alpha_{h}-d \phi_{u}^{a}(g h), v+\rho\left((g h)^{-1}\right) u, a\right) .
$$

The restriction of $\tilde{\Gamma}$ to $T^{*} G \times\{0\} \times\{a\}$ coincides with $\Gamma$. Thus, the action of Guillemin and Sternberg [1980] is a restriction of the cotangent lift of left translation for the (nonstandard) semidirect product group $\tilde{S}$, followed by an isomorphism of $S$ with $\tilde{S}$ whose induced Lie algebra isomorphism is the identity.

By Lemma 3.2, the momentum maps of the actions $\tilde{L}$ of $\tilde{S}$ and $\tilde{\Gamma}$ of $S$ on $T^{*} S$ coincide. In a symmetric way one can construct an action of $\tilde{S}$ on $T^{*} S$ with the momentum map $J_{L}$.

(3) Holmes and Marsden [1983] work with the restriction of the right action $\left(T L_{(\mathrm{g}, u)}\right)^{*}$ to $T^{*} G$ for fixed $a \in \mathrm{g}^{*}, V=\mathrm{g}, \rho=\mathrm{Ad}$.

In many physical examples a Hamiltonian system on $T^{*} G$, whose Hamiltonian function $H_{a}$ depends smoothly on a parameter $a \in V^{*}$, is given. In addition, $H_{a}$ is left invariant under the stablizer $G_{a}=\left\{g \in G \mid \rho_{*}(g) a=a\right\}$ whose Lie algebra is $\mathrm{g}_{a}=\left\{\xi \in g \mid \rho^{\prime}(\xi)^{*} a=0\right\}$. Denote by $T^{*} G / G_{a}$ the orbit space of the lift to $T^{*} G$ of left translation of $G_{a}$ on $G$. We wish to study the motion on the Poisson manifolds $T^{*} G / G_{a}$ for all $a \in V^{*}$. We shall prove below that this is equivalent to the study of the motion on $\mathfrak{s}_{-}^{*}$ where $\mathfrak{s}=\mathrm{g} \times{ }_{\rho^{\prime}} V$.

For fixed $a \in V^{*}$, the lift to $T^{*} G$ of the left translation of $G_{a}$ on $G$ has the Ad*-equivariant momentum map $J_{L}^{a}: T^{*} G \rightarrow \mathrm{g}_{a}^{*}, J_{L}^{a}\left(\alpha_{g}\right)=\left(T_{e} R_{g}\right)^{*} \alpha_{g} \mid \mathrm{g}_{a}$. The mapping $i_{L}^{a}: T^{*} G \rightarrow T^{*} S, i_{L}^{a}\left(\alpha_{g}\right)=\left(\alpha_{g}, 0, a\right)$ is an embedding of Poisson manifolds inducing a Poisson embedding of the quotients $\bar{i}_{L}^{a}: T^{*} G / G_{a} \rightarrow T^{*} S / L$. Thus, the symplectic leaves in $T^{*} S / L$ pull back to symplectic leaves in $T^{*} G / G_{a}$. The natural candidates for these leaves are the reduced symplectic manifolds $\left(J_{L}^{a}\right)^{-1}\left(\theta_{\mu_{a}}^{+}\right) / G_{a}$, where $\mu \in \mathrm{g}^{*}, \mu_{a}=\mu \mid \mathrm{g}_{a}$, and $\vartheta_{\mu_{a}}^{+} \subset \mathrm{g}_{a}^{*}$ denotes the coadjoint orbit of $G_{a}$ in $\mathrm{g}_{a}^{*}$ with the " +" Lie-Poisson symplectic structure.

For Hamiltonians that are right invariant under $G_{a}$ one proceeds exactly as above, interchanging "left" and "right", and " -" and " +", and replacing the mapping $i_{L}^{a}$ by $i_{R}^{a}\left(\alpha_{g}\right)=\left(\alpha_{g}, 0, \rho_{*}(g) a\right)$. The mappings $i_{L}^{a}$ and $i_{R}^{a}$ are necessarily different; if one 
would use $i_{L}^{a}$ for the right actions, $\bar{i}_{L}^{a}$ would fail to be injective. Summarizing: we have the following sequences of Poisson embeddings, the last arrow in each being a diffeomorphism:

$$
\begin{aligned}
& \left(J_{L}^{a}\right)^{-1}\left(\mathcal{O}_{\mu_{a}}^{+}\right) / G_{a} \hookrightarrow T^{*} G / G_{a} \stackrel{\overline{i_{L}^{u}}}{\hookrightarrow} T^{*} S / L \stackrel{\overline{J_{R}}}{\rightarrow} \mathfrak{\Im}_{-}^{*}, \\
& G_{a} \backslash\left(J_{R}^{a}\right)^{-1}\left(\mathcal{O}_{\mu_{a}}^{-}\right) \hookrightarrow G_{a} \backslash T^{*} G \stackrel{\overline{i_{R}^{u}}}{\hookrightarrow} R \backslash T^{*} S \stackrel{\overline{J_{l}}}{\rightarrow} \mathfrak{g}_{+}^{*}
\end{aligned}
$$

Moreover,

$$
\begin{aligned}
\left(\bar{J}_{R} \circ \bar{i}_{L}^{a}\right)\left(\left(J_{L}^{a}\right)^{-1}\left(\vartheta_{\mu_{a}}^{+}\right) / G_{a}\right)=\left(J_{R} \circ i_{L}^{a}\right)\left(\left(J_{L}^{a}\right)^{-1}\left(\vartheta_{\mu_{a}}^{+}\right)\right) \\
=\left\{(\nu, b) \in \mathfrak{g}^{*} \mid \text { there exists } g \in G \text { such that } \rho_{*}(g) a=b, \operatorname{Ad}_{g}^{*} \nu \in \mathcal{\Theta}_{\mu_{a}}\right\} \\
=\bigcup_{\chi \mid g_{a}=\mu_{a}} S \cdot(\chi, a)_{-}
\end{aligned}
$$

as a simple verification shows; here $S \cdot(\chi, a)_{-} \subset \mathfrak{g}_{-}^{*}$ denotes the coadjoint orbit of $S$ through $(\chi, a)$ with the " - " Lie-Poisson structure. But

$$
\left\{\left(\rho_{u}^{\prime}\right)^{*} a \mid u \in V\right\}=\left\{\mu \in \mathfrak{g}^{*}|\mu| \mathfrak{g}_{a} \equiv 0\right\}
$$

implies that $S \cdot(\chi, a)=S \cdot(\mu, a)$ for all $\chi \in \mathrm{g}^{*}$ such that $\chi \mid \mathfrak{g}_{a}=\mu_{a}$ and so we have proved the following theorem.

3.3. ThEOREM. (1) $\bar{J}_{R} \circ \bar{i}_{L}^{a}$ maps the reduced space $\left(J_{L}^{a}\right)^{-1}\left(\Theta_{\mu_{a}^{+}}\right) / G_{a}$ in a symplectically diffeomorphic way to the coadjoint orbit $S \cdot(\mu, a)_{-}$in the dual of the semidirect product $\mathfrak{\Im}_{-}^{*}$. Similarly,

(2) $\bar{J}_{L} \circ \bar{i}_{R}^{a}$ maps the reduced space $G_{a} \backslash\left(J_{R}^{a}\right)^{-1}\left(\Theta_{\mu_{u}}^{-}\right)$in a symplectically diffeomorphic way to the coadjoint orbit $S \cdot(\mu, a)_{+}$in the dual of the semidirect product $\mathfrak{\Im}_{+}^{*}$.

This result strengthens that of Ratiu $[\mathbf{1 9 8 0}, 1982]$ where the symplectic diffeomorphism $\bar{J}_{R} \circ \bar{i}_{L}^{a}$ was found and examined on an ad hoc basis. This map was shown in Holmes and Marsden [1983] to be essentially the same (except for signs, inverses and interchanges of left and right translations) as the momentum map for the action of Guillemin and Sternberg [1980]. (See also Guillemin and Sternberg [1982].) The present approach shows how the results are all obtained in a simple and natural way and unifies them with Kupershmidt's map [1982].

Next we shall explain how to use Theorem 3.3 in examples. This will allow one to begin with the standard phase space $T^{*} G$ and then to reduce a Hamiltonian system on $T^{*} G$ to a Lie-Poisson system on $\mathfrak{g}^{*}$.

Let $H_{a}: T^{*} G \rightarrow \mathbf{R}$ be a Hamiltonian depending smoothly on the parameter $a \in V^{*}$ and assume $H_{a}$ is invariant under the lift to $T^{*} G$ of the left translation of $G_{a}$ on $G$. Thus, $H_{a}$ induces a Hamiltonian on $T^{*} G / G_{a}$. Via the symplectic diffeomorphism $\bar{J}_{R} \circ \bar{i}_{L}^{a}$ we may regard it as being defined on $S \cdot(\mu, a)_{-}, \mu \in \mathrm{g}^{*}$, and now varying $a \in V^{*}$ we obtain a smooth Hamiltonian $H$ on $\mathfrak{g}_{-}^{*}$; thus $H \circ J_{R} \circ i_{L}^{a}=H_{a}$, i.e. $H\left(\left(T_{e} L_{g}\right)^{*} \alpha_{g}, \rho^{*}(g) a\right)=H_{a}\left(\alpha_{g}\right)$. Thus, the family of Hamiltonians $H_{a}$ on $T^{*} G$ induces a single Hamiltonian on $\mathfrak{s}_{-}^{*}$, and the original problem has been completely embedded in a larger one which yields Lie-Poisson equations on $\mathfrak{g}_{-}^{*}$. For right 
invariant Hamiltonians, interchange "left" and "right", and " -" and " +". However, since the maps $i_{L}^{a}$ and $i_{R}^{a}$ are different, we have $H\left(\left(T_{e} R_{g}\right)^{*} \alpha_{g}, \rho_{*}(g) a\right)=H_{a}\left(\alpha_{g}\right)$.

It is of interest to investigate the evolution of $a \in V^{*}$. Let $c_{a}(t) \in T^{*} G$ denote an integral curve of $H_{a}$ and let $g_{a}(t)$ be its projection on $G$. Then the curve $t \mapsto$ $\left(J_{R} \circ i_{L}^{a}\right) c_{a}(t)$ is an integral curve of $X_{H}$ on $\mathfrak{\Im}_{-}^{*}$ and thus $t \mapsto \rho^{*}\left(g_{a}(t)\right)$ is the evolution of the initial condition $a$ in $\mathfrak{S}_{-}^{*}$. We summarize these results in the following theorem.

3.4. TheOREM. Let $H_{a}: T^{*} G \in \mathbf{R}$ be a Hamiltonian depending smoothly on $a \in V^{*}$ and left invariant under the action on $T^{*} G$ of the stabilizer $G_{a}$. The family of Hamiltonians $\left\{H_{a} \mid a \in V^{*}\right\}$ induces a Hamiltonian function $H$ on $\mathfrak{g}_{-}^{*}$, defined by $H\left(\left(T_{e} L_{g}\right)^{*} \alpha_{g}, \rho^{*}(g) a\right)=H_{a}\left(\alpha_{g}\right)$ thus yielding Lie-Poisson equations on $\mathfrak{\xi}_{-}^{*}$. The curve $t \mapsto c_{a}(t) \in T^{*} G$ is a solution for Hamilton's equations defined by $H_{a}$ on $T^{*} G$ iff $t \mapsto\left(J_{R} \circ i_{L}^{a}\right) c_{a}(t)$ is a solution of the Hamiltonian system $X_{H}$ on $\mathfrak{s}_{-}^{*}$. In particular, the evolution of $a \in V^{*}$ is given by $t \mapsto \rho^{*}\left(g_{a}(t)\right) a$, where $g_{a}(t)$ is the projection of $c_{a}(t)$ on G. For right invariant systems, interchange everywhere "left" and "right", " -" and " +", and replace the formula for $H$ by $H\left(\left(T_{e} R_{g}\right)^{*} \alpha_{g}, \rho_{*}(g) a\right)=H_{a}\left(\alpha_{g}\right)$.

This theorem is sometimes not applied directly, since $\rho$ and $V$ may not be given but need to be discovered in the course of analyzing a system. In the process of finding $V$, one also discovers whether left or right actions of $G$ are involved. The determination of $\rho$ and $V$ usually is done by means of the evolution of $a \in V^{*}$. We shall elaborate on this remark in the context of the examples.

Finally, we remark that by shifting the reduction point to zero (see the final remarks in §1) we get the following symplectic diffeomorphisms: for a left action,

$$
\begin{aligned}
\vartheta_{\mu, a}^{-} & \approx\left(J_{L}^{a}\right)^{-1}\left(\vartheta_{\mu_{a}^{+}}^{+}\right) / G_{a} \quad \text { (by Theorem 3.3) } \\
& \approx\left(T^{*} G \ominus \vartheta_{\mu_{a}^{+}}^{+}\right)_{0} \\
& \approx\left(T^{*} G \oplus \vartheta_{\mu_{a}}^{-}\right)_{0},
\end{aligned}
$$

while for a right action,

$$
\begin{aligned}
\mathcal{O}_{(\mu, a)}^{+} & \approx G_{a} \backslash\left(J_{R}^{a}\right)^{-1}\left(\vartheta_{\mu_{a}^{-}}^{-}\right) \quad \text { (by Theorem 3.3) } \\
& \approx_{0}\left(T^{*} G \ominus \vartheta_{\mu_{a}^{-}}^{-}\right) \\
& \approx_{0}\left(T^{*} G \oplus \vartheta_{\mu_{a}}^{+}\right) .
\end{aligned}
$$

The reduced spaces like $\left(T^{*} G \ominus \mathcal{O}_{\mu_{u}}^{+}\right)_{0}$ are examples of spaces of the type considered in Weinstein [1978].

4. Clebsch variables. A general framework for symplectic (or "Clebsch") variables was described in Marsden and Weinstein [1982b]. We recall that if $(P,\{\}$,$) is a$ Poisson manifold, then by symplectic variables for $P$ we mean a Poisson map $J$ : $M \rightarrow P$, where $M$ is a symplectic manifold. If canonical coordinates are chosen on $M$, we call them canonical variables for $P$. A Hamiltonian $H: P \rightarrow \mathbf{R}$ determines one on $M$ by $H_{M}=H \circ J$, and the integral curves for the standard (or "canonical") 
Hamiltonian system $H_{M}$ on $M$ cover those for the Poisson (or "noncanonical") Hamiltonian system $H$ on $P$. If $P=\mathrm{g}_{+}^{*}$ then $J$ is necessarily the left momentum map for an action of $G$ on $M$; similarly for right actions and $g_{-}^{*}$.

In our case, we choose $P=\mathfrak{S}_{ \pm}^{*}$, the " \pm " Lie-Poisson space for the semidirect product $S=G \times{ }_{\rho} V$. One choice of Clebsch variables is simply $T^{*} S=T^{*} G \times V \times$ $V^{*}$, with $J_{L}$ and $J_{R}$ given in the previous section. In some circumstances, though, it is useful to use a "flat" space. We shall give an abstract construction which includes the classical Clebsch representation for the momentum density of a compressible fluid (see $\$ 5$ for a description of this example).

The construction is very simple. The semidirect product $S=G \times V$ acts on $V \times V^{*} \times V \times V^{*}$ by

$$
(g, u) \cdot((v, a),(w, b))=\left(\rho(g) v, \rho_{*}(g) a, u+\rho(g) w, \rho_{*}(g) b\right)
$$

which is the cotangent lift of the left action of $S$ on $V \times V$ by the affine transformations

$$
(g, u) \cdot(v, w)=(\rho(g) v, u+\rho(g) w) .
$$

The corresponding momentum map is

$$
\langle J(v, a, w, b),(\xi, u)\rangle=\left\langle a, \rho^{\prime}(\xi) \cdot v\right\rangle+\left\langle b, \rho^{\prime}(\xi) \cdot w\right\rangle+\langle b, u\rangle .
$$

Therefore,

$$
J(v, a, w, b)=\left(\rho_{v}^{\prime *} a+\rho_{w}^{*} b, b\right)
$$

and $J$ is a Poisson map of $V \times V^{*} \times V \times V^{*}$ to $\mathfrak{\Im}_{+}^{*}$.

The procedure above will be related to the Clebsch expression $M=\rho \nabla \phi+\lambda \nabla \mu$ for compressible fluids in Example 5.B. For incompressible fluids, there is a closely related construction described in Marsden and Weinstein [1982b], leading to the Clebsch representation $\omega=d \lambda \wedge d \mu$ for the vorticity. We refer the reader to this reference for further discussion, examples and limitations.

The construction above can be simplified to find a Poisson map $J: V \times V^{*} \rightarrow \mathfrak{g}_{+}^{*}$; this will be related to the Clebsch representation for magnetohydrodynamics in Example 5.C (see Morrison [1982], Holm and Kupershmidt [1982]). Let $S$ act on $V$ by

$$
(g, u) \cdot w=u+\rho(g) w .
$$

The lift of this action to $V \times V^{*}$ is

$$
(g, u) \cdot(v, a)=\left(u+\rho(g) v, \rho_{*}(g) a\right)
$$

which has its momentum mapping $J: V \times V^{*} \rightarrow \mathfrak{g}_{+}^{*}$ given by

$$
J(v, a)=\left(\rho_{v}^{* *} a, a\right) .
$$

In some applications, the semidirect product is of the form $G \times V \times W$, where $\rho$ : $G \rightarrow \operatorname{Aut}(V), \sigma: G \rightarrow \operatorname{Aut}(W)$ are left representations. Then we can get a Poisson map

$$
\begin{aligned}
& J: V \times V^{*} \times V \times V^{*} \times W \times W^{*} \rightarrow \mathfrak{S}_{+}^{*}, \\
& J(u, a, v, b, w, c)=\left(\rho_{v}^{\prime *} a+\rho_{v}^{\prime *} b+\sigma_{w}^{\prime *} c, b, c\right) .
\end{aligned}
$$


This is the momentum map of the lift to the cotangent bundle of the left action of $S$ on $V \times V \times W$ given by

$$
(g, v, w) \cdot\left(u_{1}, u_{2}, z\right)=\left(\rho(g) u_{1}, v+\rho(g) u_{2}, w+\sigma(g) z\right) .
$$

This construction gives the Clebsch representation of isentropic fluid flow and magnetohydrodynamics when entropy is present, as we shall see in Examples 5.B and 5.C.

5. Examples. We shall treat four physical examples: the heavy top, compressible fluids, ideal magnetohydrodynamics and elasticity. We shall not spare details in presenting the first two examples, to help the reader put the basic principles into our setting.

A. The heavy top. We recall a few facts about the rotation group $\mathrm{SO}(3)$ and its Lie algebra so(3), the set of $3 \times 3$ skew symmetric matrices. We identify so(3) with $\mathbf{R}^{3}$ by identifying

$$
\boldsymbol{x}=(p, q, r) \quad \text { with } \hat{\boldsymbol{x}}=\left[\begin{array}{ccc}
0 & -r & q \\
r & 0 & -p \\
-q & p & 0
\end{array}\right]
$$

thus, $\hat{x} \boldsymbol{y}$ equals the cross product $\boldsymbol{x} \times \boldsymbol{y}$ for $\boldsymbol{x}$ and $\boldsymbol{y}$ in $\mathbf{R}^{3}$. The Lie algebra bracket is then $[\hat{x}, \hat{y}]=(\boldsymbol{x} \times \boldsymbol{y})^{\hat{y}}$. The adjoint action of $\operatorname{SO}(3)$ on $\operatorname{so}(3)$ corresponds to the usual action of $\operatorname{SO}(3)$ in $\mathbf{R}^{3}$, i.e. $\operatorname{Ad}_{A} \hat{x}=(A x), A \in \operatorname{SO}(3)$. We also identify the dual $\operatorname{so}(3)^{*}$ with $\mathbf{R}^{3}$ by the inner product in $\mathbf{R}^{3}$, i.e. $\tilde{\boldsymbol{m}} \in \operatorname{so}(3)^{*}$ corresponds to $\boldsymbol{m} \in \mathbf{R}^{3}$ by $\tilde{\boldsymbol{m}}(\hat{\boldsymbol{x}})=\boldsymbol{m} \cdot \boldsymbol{x}$ for $\hat{\boldsymbol{x}} \in \operatorname{so}(3)$. Then the coadjoint action of $\operatorname{SO}(3)$ on so(3)* corresponds to the usual action of $\operatorname{so}(3)$ on $\mathbf{R}^{3}$, i.e. $\operatorname{Ad}_{A^{-1}}^{*} \tilde{\boldsymbol{m}}=\widetilde{\boldsymbol{A m}}$ since $\left(A^{-1}\right)^{*}=A$.

A top is, by definition, a rigid body moving about a fixed point, which we take to be the origin of the standard coordinate system on $\mathbf{R}^{3}$. Rigidity means that the distances between points of the body are fixed when the body moves; this implies that if $f(t, \boldsymbol{x})$ is the position at time $t$ of the particle that was at $\boldsymbol{x}$ at time $t=0$, we have $f(t, \boldsymbol{x})=\boldsymbol{A}(t) \boldsymbol{x}$, where $\boldsymbol{A}(t)$ is an orthogonal matrix. Since the motion is assumed to be continuous and $\boldsymbol{A}(0)=\mathrm{Id}=$ the identity matrix, $\operatorname{det} \boldsymbol{A}(t)>0$ and so $A(t) \in \mathrm{SO}(3)$. Thus the configuration space of the rigid body may be identified with $\mathrm{SO}(3)$.

The initial mass distribution of the body is described by a positive measure $\mu$ on $\mathbf{R}^{3}$ which is supported on a compact set with nonempty interior. The kinetic energy at time $t$ is

$$
K(t)=\frac{1}{2} \int_{\mathbf{R}^{3}}\|\dot{f}(t, \boldsymbol{x})\|^{2} d \mu(\boldsymbol{x})=\frac{1}{2} \int_{\mathbf{R}^{3}}\|\dot{\boldsymbol{A}}(t) \boldsymbol{x}\|^{2} d \mu(\boldsymbol{x}) .
$$

This can be written as

$$
\frac{1}{2}\langle\hat{\boldsymbol{\omega}}(t), \hat{\boldsymbol{\omega}}(t)\rangle=\frac{1}{2} \boldsymbol{I} \boldsymbol{\omega}(t) \cdot \boldsymbol{\omega}(t)
$$

where $\hat{\omega}(t)=\boldsymbol{A}(t)^{-1} \dot{A}(t) \in \operatorname{so}(3)$ is the angular velocity in the body, $\langle\xi, \eta\rangle$ is the inner product on so(3) defined by $\int_{\mathbf{R}^{3}} \xi \boldsymbol{x} \cdot \boldsymbol{\eta} \boldsymbol{x} d \mu(\boldsymbol{x})$, and $\boldsymbol{I}: \mathbf{R}^{3} \rightarrow \mathbf{R}^{3}$ is the symmetric 
operator determined by $\langle\hat{\boldsymbol{x}}, \hat{\boldsymbol{y}}\rangle=\boldsymbol{I} \boldsymbol{x} \cdot \boldsymbol{y} . \boldsymbol{I}$ is called the moment of inertia tensor. The basis in which it is diagonal is the principal axis body frame.

The standard phase space for the rigid problem is $T^{*} \mathrm{SO}(3)$ (Euler angles and their conjugate momenta). The inner product $\langle$,$\rangle on \operatorname{so}(3)$ induces a norm on $\operatorname{so}(3)^{*}$ and by left translation a length function \|\| on $T^{*} \operatorname{SO}(3)$. If $I=\operatorname{diag}\left(I_{1}, I_{2}, I_{3}\right), \boldsymbol{m}=$ $\left(T_{\mathrm{Id}} L_{A}\right)^{*} \alpha_{A}$, and $\alpha_{A} \in T_{A}^{*} \mathrm{SO}(3)$, the kinetic energy has two alternate expressions:

$$
K=\frac{1}{2}\left\|\alpha_{A}\right\|^{2} \text { as a function on } T^{*} \mathrm{SO}(3)
$$

or

$$
K=\frac{1}{2} \boldsymbol{m} \cdot \boldsymbol{I}^{-1} \boldsymbol{m}=\frac{1}{2}\left(\frac{m_{1}^{2}}{I_{1}}+\frac{m_{2}^{2}}{I_{2}}+\frac{m_{3}^{2}}{I_{3}}\right) \text { as a function on } \mathbf{R}^{3} \cong \operatorname{so}(3)^{*} \text {. }
$$

The potential energy $V$ for a heavy top is determined by the height of the center of mass over a horizontal plane in the spatial coordinate system. Let $l \boldsymbol{X}$ denote the vector determining the center of mass in the body frame at $t=0$, where $\boldsymbol{X}$ is a unit vector along the straight line segment of length $l$ connecting the fixed point with the center of mass. Thus, if $M=\int_{\mathbf{R}^{3}} d \mu(\boldsymbol{x})$ is the total mass of the body, $g$ is the gravitational acceleration, and $\boldsymbol{k}$ denotes the unit vector along the spatial $O z$-axis, the potential energy at time $t$ is

$$
V(t)=M g k \cdot A(t) l \boldsymbol{\chi}=M g l A^{-1} k \cdot \chi=M g l v \cdot \chi
$$

where $\boldsymbol{v}=\boldsymbol{A}^{-1} \boldsymbol{k}$. The potential energy is

$$
\begin{array}{ll}
M g l \boldsymbol{A}^{-1} \boldsymbol{k} \cdot \boldsymbol{\chi} & \text { as a function on } \mathrm{SO}(3), \\
M g l \boldsymbol{v} \cdot \boldsymbol{\chi} & \text { as a function on } \mathbf{R}^{3} .
\end{array}
$$

First we apply Theorem 3.4 directly. The Hamiltonian

$$
H_{a}\left(\alpha_{A}\right)=\frac{1}{2}\left\|\alpha_{A}\right\|^{2}+M g l A^{-1} k \cdot \chi
$$

for $\boldsymbol{a}=M g l \boldsymbol{k} \in \mathbf{R}^{3} \cong\left(\mathbf{R}^{3}\right)^{*}$ is left invariant under rotations about the spatial $\mathrm{Oz}$-axis with corresponding conserved quantity $\boldsymbol{m} \cdot \boldsymbol{v}$, the momentum about $\mathrm{Oz}$. Changing the parameter $\boldsymbol{a}=M g l \boldsymbol{k}$ corresponds to changing the magnitude of the momentum $\mathrm{Mgl}$ of the body about the fixed point and the direction $\boldsymbol{k}$ of the observer. We take the representation $\rho: \mathrm{SO}(3) \rightarrow \operatorname{Aut}\left(\mathbf{R}^{3}\right)$ to be the standard one: $\rho(\boldsymbol{A}) \boldsymbol{x}=\boldsymbol{A} \boldsymbol{x}$. By Theorem 3.4 we get a Hamiltonian

$$
H(\boldsymbol{m}, \boldsymbol{v})=H\left(\left(T_{I d} L_{\boldsymbol{A}}\right)^{*} \alpha_{\boldsymbol{A}}, \rho^{*}(\boldsymbol{A}) \boldsymbol{k}\right)=H_{a}\left(\alpha_{\boldsymbol{A}}\right)=\frac{1}{2} \boldsymbol{m} \cdot \boldsymbol{I}^{-1} \boldsymbol{m}+M g \boldsymbol{v} \cdot \boldsymbol{\chi}
$$

defining the Lie-Poisson equations on the dual of the Euclidean Lie algebra $e(3)$.

We can also apply Theorem 3.4 backwards, starting from the variables $\boldsymbol{m}, \boldsymbol{v}$ in the Hamiltonian. The configuration space is $\mathrm{SO}(3)$, and the Hamiltonian $H(\boldsymbol{m}, \boldsymbol{v})=$ $\frac{1}{2} \boldsymbol{m} \cdot \boldsymbol{I}^{-1} \boldsymbol{m}+M g l \boldsymbol{v} \cdot \boldsymbol{\chi}$ is defined in $\mathbf{R}^{3} \times \mathbf{R}^{3} \cong \operatorname{so}(3)^{*}\left(\mathbf{R}^{3}\right)^{*}$. We also take as given the fact that $\boldsymbol{v}=\boldsymbol{A}^{-1} \boldsymbol{k}$. Since $\boldsymbol{A} \mapsto \boldsymbol{A}^{-1} \boldsymbol{k}$ is a right representation describing the time evolution of the parameter $\boldsymbol{a}$, by Theorem 3.4 we must look for a representation $\rho$ such that $\rho^{*}(\boldsymbol{A}) \boldsymbol{u}=\boldsymbol{A}^{-1} \boldsymbol{u}$ for any $\boldsymbol{u} \in\left(\mathbf{R}^{3}\right)^{*}$. This uniquely determines $\rho(\boldsymbol{A}) \boldsymbol{x}=\boldsymbol{A} \boldsymbol{x}$ and defines the Hamiltonian $H_{a}\left(\alpha_{A}\right)=H\left(\left(T_{\mathrm{Id}} L_{\boldsymbol{A}}\right)^{*} \alpha_{A}, \rho^{*}(\boldsymbol{A}) \boldsymbol{k}\right)=\frac{1}{2}\left\|\alpha_{A}\right\|^{2}+$ $M g l \boldsymbol{A}^{-1} \boldsymbol{k} \cdot \boldsymbol{X}$. Now by Theorem 3.4 we conclude again that $H(\boldsymbol{m}, \boldsymbol{v})$ determines the Lie-Poisson equations in $e(3)^{*}$. 
To write the bracket and the Lie-Poisson equations explicitly, we note first that $\rho^{\prime}$ : $\operatorname{so}(3) \rightarrow \operatorname{End}\left(\mathbf{R}^{3}\right)$ is given by $\rho^{\prime}(\xi) \boldsymbol{x}=\boldsymbol{\xi} \boldsymbol{x}$, where $\boldsymbol{\xi} \in \operatorname{so}(3)$ and $\boldsymbol{x} \in \mathbf{R}^{3}$. For $F, G$ : $e(3)^{*} \rightarrow \mathbf{R}, \delta F / \delta \boldsymbol{m}=\left(\nabla_{\boldsymbol{m}} F\right)^{\hat{n}}, \delta F / \delta \boldsymbol{v}=\nabla_{\boldsymbol{v}} F\left(\nabla_{\boldsymbol{m}}, \nabla_{\boldsymbol{v}}\right.$ denote the usual gradients with respect to $\boldsymbol{m}, v \in \mathbf{R}^{3}$ ) and, hence,

$$
\begin{gathered}
\rho^{\prime}\left(\frac{\delta F}{\delta \boldsymbol{m}}\right) \cdot \frac{\delta G}{\delta \boldsymbol{v}}=\left(\nabla_{\boldsymbol{m}} F\right)^{\wedge} \nabla_{\boldsymbol{v}} G=\nabla_{\boldsymbol{m}} F \times \nabla_{\boldsymbol{v}} G, \\
\rho^{\prime}\left(\frac{\delta F}{\delta \boldsymbol{m}}\right)^{*} \cdot v=-\nabla_{\boldsymbol{m}} F \times \boldsymbol{v}, \quad\left(\rho_{\delta F / \delta v}^{\prime}\right)^{*} v=\nabla_{v} F \times v,
\end{gathered}
$$

and

$$
\operatorname{ad}\left(\frac{\delta F}{\delta \boldsymbol{m}}\right)^{*} \cdot \boldsymbol{m}=-\nabla_{\boldsymbol{m}} F \times \boldsymbol{m} .
$$

The Lie-Poisson equations (3.2) become, for this case,

$$
\left\{\begin{array}{l}
\dot{\boldsymbol{m}}=-\nabla_{\boldsymbol{m}} H \times \boldsymbol{m}-\nabla_{\boldsymbol{v}} H \times \boldsymbol{v}, \\
\dot{\boldsymbol{v}}=-\nabla_{\boldsymbol{m}} H \times \boldsymbol{v},
\end{array}\right.
$$

or, explicitly taking into account that $\nabla_{\boldsymbol{m}} H=\left(m_{1} / I_{1}, m_{2} / I_{2}, m_{3} / I_{3}\right)$ and $\nabla_{\boldsymbol{v}} H=$ $M g l \chi$

$$
\begin{aligned}
& \begin{cases}\dot{m}_{1}=a_{1} m_{2} m_{3}+\operatorname{Mgl}\left(\chi_{3} v_{2}-\chi_{2} v_{3}\right), & a_{1}=\left(1 / I_{3}\right)-\left(1 / I_{2}\right), \\
\dot{m}_{2}=a_{2} m_{1} m_{3}+\operatorname{Mgl}\left(\chi_{1} v_{3}-\chi_{3} v_{1}\right), & a_{2}=\left(1 / I_{1}\right)-\left(1 / I_{3}\right), \\
\dot{m}_{3}=a_{3} m_{1} m_{2}+M g l\left(\chi_{2} v_{1}-\chi_{1} v_{2}\right), & a_{3}=\left(1 / I_{2}\right)-\left(1 / I_{1}\right),\end{cases} \\
& \left\{\begin{array}{l}
\dot{v}_{1}=m_{3} v_{2} / I_{3}-m_{2} v_{3} / I_{2}, \\
\dot{v}_{2}=m_{1} v_{3} / I_{1}-m_{3} v_{1} / I_{3}, \\
\dot{v}_{3}=m_{2} v_{1} / I_{2}-m_{1} v_{2} / I_{1} .
\end{array}\right.
\end{aligned}
$$

Finally, the Lie-Poisson bracket on $e(3)_{-}^{*}$ of two functions $F$ and $G$ is, by (3.1),

$$
\{F, G\}_{-}(\boldsymbol{m}, \boldsymbol{v})=-\boldsymbol{m} \cdot\left(\nabla_{\boldsymbol{m}} F \times \nabla_{\boldsymbol{m}} G\right)-\boldsymbol{v} \cdot\left(\nabla_{\boldsymbol{m}} F \times \nabla_{\boldsymbol{v}} G+\nabla_{\boldsymbol{v}} F \times \nabla_{\boldsymbol{m}} G\right) .
$$

Writing $\dot{F}=\{F, H\}_{-}$, for $F=m_{1}, m_{2}, m_{3}, v_{1}, v_{2}, v_{3}$, directly produces the prior Lie-Poisson equations.

For the explicit formulas relating the Lie-Poisson variables $(\boldsymbol{m}, \boldsymbol{v})$ to the Euler angles and their conjugate momenta, and a direct verification of the equivalence of the two descriptions, see Holmes and Marsden [1983].

We shall now find Clebsch variables for the heavy top. The simplest Poisson map is given by (4.2), namely $J: \mathbf{R}^{3} \times \mathbf{R}^{3} \rightarrow \mathbf{R}^{3} \times \mathbf{R}^{3}\left(\cong e(3)_{+}^{*}\right), J(\boldsymbol{x}, \boldsymbol{v})=\left(\rho_{x}^{\prime *} \boldsymbol{v}, \boldsymbol{v}\right)=(\boldsymbol{x}$ $\times \boldsymbol{v}, \boldsymbol{v})$. The trouble with this map is that it maps onto a codimension one submanifold of $\mathbf{R}^{3} \times \mathbf{R}^{3}$, since $\boldsymbol{x} \times \boldsymbol{v}$ is always orthogonal to $\boldsymbol{v}$. Formula (4.1) gives a Poisson map $J:\left(\mathbf{R}^{3}\right)^{4} \rightarrow \mathbf{R}^{3} \times \mathbf{R}^{3}, J(x, \boldsymbol{u}, \boldsymbol{y}, \boldsymbol{v})=\left(\rho_{x}^{\prime *} \boldsymbol{u}+\rho_{y}^{\prime} \boldsymbol{v}, \boldsymbol{v}\right)=(\boldsymbol{x} \times \boldsymbol{u}+\boldsymbol{y} \times$ $\boldsymbol{v}, \boldsymbol{v})$ which is onto. If we let $\bar{H}(\boldsymbol{x}, \boldsymbol{y}, \boldsymbol{y}, \boldsymbol{v})=H(\boldsymbol{x} \times \boldsymbol{u}+\boldsymbol{y} \times \boldsymbol{v}, \boldsymbol{v})$, then the heavy top equations have the canonical form

$$
\dot{x}_{i}=-\partial H / \partial u_{i}, \quad \dot{u}_{i}=\partial H / \partial x_{i}, \quad \dot{y}_{i}=-\partial H / \partial v_{i}, \quad \dot{v}_{i}=\partial H / \partial y_{i} .
$$

The signs in Hamilton's equations are opposite to the usual ones, since $J$ maps onto $e(3)_{+}^{*}$, whereas the heavy top equations live on $e(3)_{-}^{*}$. 
One might argue that $\mathbf{R}^{12}$ is too large. This is indeed true, and a theorem of Lie [1890] assures that the minimal-dimensional symplectic manifold on which $E(3)$ acts freely is 8-dimensional. Using a global version of this theorem in Weinstein [1982], one can show that one such manifold is $E(3) \times \mathbf{R}^{2}$ with $E(3)$ acting only on the first factor. The symplectic structure is quite twisted, so we will not elaborate on it any further.

B. Ideal compressible isentropic fluids. ${ }^{4}$ Let $\Omega$ be a compact submanifold of $\mathbf{R}^{3}$ with smooth boundary, filled with a moving fluid free of exterior forces. Denote by $x(t)=\eta_{t}(X)=\eta(X, t)$, where $X \in \Omega$, the trajectory of a fluid particle which at time $t=0$ is at $X$. As is customary in continuum mechanics, capital letters will denote entities in the reference configuration, i.e., in "body" coordinates; lower case letters denote spatial entities (see Marsden and Hughes [1983]). Given $\eta_{t}: \Omega \rightarrow \Omega$, a time dependent diffeomorphism of $\Omega$, denote by $v_{t}(x)=v(x, t)$ the spatial velocity field of the fluid, i.e.

$$
\frac{\partial \eta(X, t)}{\partial t}=v(\eta(X, t), t)
$$

$v_{t}$ is thus a time dependent vector field with flow $\eta_{t}$. Let $\rho_{t}(x)=\rho(x, t)$ denote the mass density of the fluid at time $t$, and $\rho_{0}$ the mass density in the reference configuration. Thus the physical problem of fluid motion has as configuration space the group $\omega_{2}$ of diffeomorphisms of $\Omega$, and $\rho_{t}$ is determined by the configuration when $\rho_{0}$ is known.

The equations of motion are derived from three fundamental principles: conservation of mass, momentum and energy. It will be useful to recall these well-known derivations, as they are relevant to understanding how Theorem 3.4 is applied.

(a) The principle of conservation of mass stipulates that mass can be neither created or destroyed, i.e.

$$
\int_{\eta_{t}(W)} \rho_{t}(x) d x=\int_{W} \rho_{0}(X) d X
$$

for all compact $W$ with nonempty interior having smooth boundary. Changing variables, this becomes

$$
\eta_{t}^{*}\left(\rho_{t}(x) d x\right)=\rho_{0}(X) d X
$$

or

$$
\left(\eta_{t}^{*} \rho_{t}\right) J\left(\eta_{t}\right)=\rho_{0}
$$

where $J\left(\eta_{t}\right)=d x / d X$ is the Jacobian of $\eta_{t}$. Using the relation between Lie derivatives and flows, this is equivalent to the continuity equation

$$
\partial \rho / \partial t+\operatorname{div}\left(\rho_{t} v\right)=0
$$

The present derivation of conservation of mass shows that the physical entity to be dealt with is the density $\rho d x$ rather than the function $\rho$ and that (5.1) or (5.2) is more

\footnotetext{
${ }^{4}$ For expository reasons, technical details on function spaces are omitted. See Ebin and Marsden [1970] for what is needed. The velocity fields are at least $C^{\prime}$.
} 
convenient than the standard fluid mechanics formulation (5.3). This observation will be crucial later on.

(b) The balance of momentum is described by Newton's second law: the rate of change of momentum of a portion of the fluid equals the total force applied to it. Since we assume that no external forces are present, the only forces acting on the fluid are forces of stress. The assumption of an ideal fluid means that the force of stress per unit area exerted across a surface element at $x$, with outward unit normal $n$ at time $t$, is $-p(x, t) n$ for some function $p(x, t)$ called the pressure. With this hypothesis, the balance of momentum becomes Euler's equations of motion

$$
\frac{\partial v}{\partial t}+(v \cdot \nabla) v=-\frac{1}{\rho} \nabla p
$$

with the boundary condition $v \| \partial \Omega$ (no friction exists between fluid and boundary) and the initial condition $v(x, 0)=v_{0}(x)$ on $\Omega$.

(c) The kinetic energy of the fluid is $\frac{1}{2} \int_{\Omega} \rho\|v\|^{2} d x$. The assumption of an isentropic fluid means that the internal energy of the fluid is $\int_{\Omega} \rho w(\rho) d x$ and $p=\rho^{2} w^{\prime}(\rho)$ where $p^{\prime}(\rho)>0$. These hypotheses imply that the total energy, which should be the Hamiltonian of the system, is conserved.

The configuration space of this problem is $\mathscr{D}$, and so the corresponding phase space is $T^{* \mathscr{D}}$. For $\eta \in \mathscr{D}$ we have $T_{\eta}^{\mathscr{D}}=\left\{\boldsymbol{V}_{\eta}: \Omega \rightarrow T \Omega \mid V(X) \in T_{\eta(X)} \Omega\right\}$ and $T_{\eta}^{*} \mathscr{D}$ $=\left\{\alpha_{\eta}: \Omega \rightarrow T^{*} \Omega \otimes \Lambda^{3}(\Omega) \mid \alpha(X) \in T_{\eta(X)}^{*} \Omega \otimes \Lambda_{X}^{3}(\Omega)\right\} ;$ the pairing between the velocity $V$ in $T_{\eta}^{\mathscr{D}}$ and the momentum density $\alpha$ in the dual space $T^{*} \mathscr{D}$ is

$$
\langle\alpha, V\rangle=\int_{\Omega} \alpha(X) \cdot V(X) .
$$

For later use, we shall express the energy on $T^{*} \mathscr{D}$ by passing to material coordinates. Let $V_{t}(X)=d \eta(X, t) / d t$ be the material velocity. Then $V_{t}=v_{t} \circ \eta_{t}$, showing that $V_{t}$ is not a vector field but an element of $T_{\eta_{t}} \mathscr{D}$. The metric on $\Omega$ and the density $\rho_{0}(X) d x$ establish an isomorphism of $T_{\eta}^{\mathscr{Q}}$ with $T_{\eta}^{*} \mathscr{D}$ given by $V_{\eta}(X) \mapsto \alpha_{\eta}(X)=$ $\rho_{0}(X) V_{\eta}^{b}(X) d X$ where b: $T \Omega \rightarrow T^{*} \Omega$ is the bundle isomorphism induced by the metric on $\Omega$. Finally, $\mathscr{Q}$ has a smooth metric defined by

$$
\left\langle\left\langle\boldsymbol{V}_{\eta}, \boldsymbol{W}_{\eta}\right\rangle\right\rangle_{\eta}=\int_{\Omega} \boldsymbol{V}_{\eta}(X) \cdot \boldsymbol{W}_{\eta}(X) \rho_{0}(X) d X
$$

which determines a length function on $T^{*} \mathscr{D}$, denoted by \|\| .

To apply Theorem 3.4 directly, use a simple change of variables to express the energy on $T^{*} \mathscr{Q}$ as

$$
H_{\rho_{0}}\left(\alpha_{\eta}\right)=\frac{1}{2}\left\|\alpha_{\eta}\right\|^{2}+\int_{\Omega} \rho_{0}(X) w\left(\rho_{0}(X) J_{\eta}^{-1}(X)\right) d X .
$$

$H_{\rho_{0}}$ clearly depends smoothly on $\rho_{0}$, and again the change of variables formula shows that it is right invariant under the action of the subgroup

$$
\mathscr{D}_{\rho_{0}}=\left\{\phi \in \mathscr{D} \mid \rho_{0}=\left(\rho_{0} \circ \phi^{-1}\right) J_{\phi^{-1}}\right\}
$$

where $J_{\phi}$ denotes the Jacobian of $\phi$. This shows that we should work with the density $\rho_{0}(X) d X$ and not with the function $\rho_{0}$, and consider the representation of $\mathscr{D}$ on 
$\bar{F}(\Omega)$ to be the push-forward, i.e. $(\eta, f) \mapsto \eta_{*} f$. Then the induced left representation on $\bar{r}(\Omega)^{*}=$ densities on $\Omega$, is again push-forward and

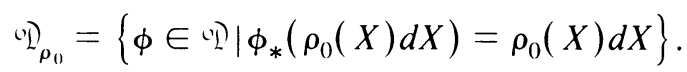

Let $S=\mathscr{Q}) \times \widetilde{\mathscr{Y}}(\Omega)$ be the semidirect product of $\mathscr{D})$ with $\widetilde{\mathscr{F}}(\Omega)$ by the push-forward representation and $s=\mathfrak{Y} \times \widetilde{\mathscr{Y}}(\Omega)$ its Lie algebra. By Theorem 3.4 for right invariant systems the family of Hamiltonians $H_{\rho_{0}}$ determines a unique Hamiltonian system on $\breve{s}_{+}^{*}$. If $\overline{\boldsymbol{M}}=\left(T_{e} R_{\eta}\right)^{*} \alpha_{\eta} \in T_{e}^{*(\mathfrak{l})}=\mathfrak{X}^{*}=$ one-form densities, for every $W \in \mathfrak{X}$,

$$
\begin{aligned}
\langle\overline{\boldsymbol{M}}, \boldsymbol{W}\rangle & =\int_{\Omega} \boldsymbol{\alpha}_{\eta}(X) \cdot \boldsymbol{W}\left(\eta(X) d X=\int_{\Omega} \rho_{0}(X) \boldsymbol{V}_{\eta}(X) \cdot \boldsymbol{W}(\eta(X)) d X\right. \\
& =\int_{\Omega} \rho(x) \boldsymbol{v}(x) \cdot \boldsymbol{W}(x) d x,
\end{aligned}
$$

i.e. $\overline{\boldsymbol{M}}=\rho(x) \boldsymbol{v}^{\mathrm{b}}(x) d x$. Thus the Hamiltonian $H$ on $\mathfrak{s}_{+}^{*}$ has the expression

$$
\begin{aligned}
H(\overline{\boldsymbol{M}}, \rho(x) d x) & =H\left(\left(T_{e} R_{\eta}\right)^{*} \boldsymbol{\alpha}_{\eta}, \eta_{*}\left(\rho_{0}(X) d X\right)\right) \\
& =H_{\rho_{0}}\left(\boldsymbol{\alpha}_{\eta}\right)=\frac{1}{2} \int_{\Omega} \rho(x)\|\boldsymbol{v}(x)\|^{2} d x+\int \rho(x) w(\rho)(x) d x
\end{aligned}
$$

which is the physical energy function. Thus, identifying $\overline{\boldsymbol{M}}$ with the momentum density $\boldsymbol{M}(x)=\rho(x) \boldsymbol{v}(x)$ and $\rho(x) d x$ with $\rho(x)$, the physical energy function

$$
H(\boldsymbol{M}, \rho)=\frac{1}{2} \int_{\Omega} \frac{1}{\rho(x)}\|\boldsymbol{M}(x)\|^{2} d x+\int_{\Omega} \rho(x) w(\rho)(x) d x
$$

defines Lie-Poisson equations on $(\mathfrak{X} \times \widetilde{\mathcal{Y}}(\Omega))_{+}^{*}$; we shall determine them later on.

We now apply Theorem 3.4 backwards. Start with the configuration space $\mathscr{D}$, the physical energy function $H(\boldsymbol{M}, \rho)$, conservation of mass (5.3), balance of momentum (5.4) and $p=\rho^{2} w^{\prime}(\rho)$. Notice that (5.3) is equivalent to $L_{v}(\rho(x) d x)=0$, i.e. $\eta_{t}^{*}(\rho(x) d x)=\rho_{0}(X) d X$, for $\rho_{0}$ the initial mass density. Hence, the dual of the representation space is the space of densities, i.e. the representation space is $\mathscr{F}(\Omega)$. Moreover, $\eta_{t^{*}}\left(\rho_{0}(X) d X\right)=\rho(x) d X$ shows that the induced left representation $\mathscr{F}(\Omega)^{*}$ is push-forward, so that, by the general formula on the evolution of the parameter $a \in V^{*}$ in Theorem 3.4, the representation of $\mathcal{Q}$ on $\widetilde{\mathcal{F}}(\Omega)$ is also push-forward. We now identify $\boldsymbol{M}$ with $\overline{\boldsymbol{M}}$ and $\rho(x)$ with $\rho(x) d x$, so that the physical energy function $H(\overline{\boldsymbol{M}}, \rho(x) d x)$ is defined on $\mathfrak{X}^{*} \times \widehat{\mathscr{Y}}(\Omega)^{*}$. Since $H(\overline{\boldsymbol{M}}, \rho(x) d x)=H_{\rho_{0}}\left(\alpha_{\eta}\right)$ for $\left(T_{e} R_{\eta}\right)^{*} \alpha_{\eta}=\overline{\boldsymbol{M}}, \eta_{*}\left(\rho_{0}(X) d X\right)=\rho(x) d x$, and $H_{\rho_{0}}$ is right invariant under $\mathscr{Q}_{\rho_{0}}$, Theorem 3.4 for right invariant systems can be applied yielding Lie-Poisson equations on $\mathfrak{s}_{+}^{*}$.

Having seen how Theorem 3.4 was applied backwards in the heavy top problem and in ideal compressible hydrodynamics, let us make some general remarks. In many examples one is given the phase space $T^{*} G$, but it is not obvious a priori what the space $V$ and the representation $\rho$ should be. The phase space $T^{*} G$ is often interpreted as 'material' or 'Lagrangian' coordinates, while the equations of motion may be partially or wholly derived in 'spatial' or 'Eulerian' coordinates. This means that the Hamiltonian might be given directly on a space of the form $\mathrm{g}^{*} \times V^{*}$, where 
the evolution of the $V^{*}$ variable is by 'dragging along' or 'Lie transport' i.e. it is of the form $t \mapsto \rho^{*}(g(t)) a$ for left invariant systems (or $t \mapsto \rho_{*}(g(t)) a$ for right invariant ones), where $a \in V^{*}$ and $g(t)$ is the solution curve in the configuration space $G$. This then determines the representation $\rho$ and shows whether one should work with left or right actions. The relation between $H$ and $H_{a}$ in the theorem uniquely determines $H_{a}$, which is automatically $G_{a}$-invariant, and (3.1) and (3.2) give the corresponding Lie-Poisson bracket and equations of motion. The parameter $a \in V^{*}$ often appears in the form of an initial condition on some physical variable of the given problem.

Let us now return to ideal compressible isentropic fluids. To write the bracket and the Lie-Poisson equations explicitly, note first that the induced Lie algebra representation $\rho^{\prime}: \mathfrak{X} \rightarrow \operatorname{End}(\mathscr{F}(\Omega))$ is given by $\rho^{\prime}(v) f=-L_{v} f$, where $L_{v}$ is the Lie derivative. To see this, denote by $\eta_{t}$ the flow of $v$ and obtain

$$
\rho^{\prime}(v) f=\left.\frac{d}{d t}\right|_{t=0} \rho\left(\eta_{t}\right) f=\left.\frac{d}{d t}\right|_{t=0}\left(\eta_{t}\right)_{*} f=\left.\frac{d}{d t}\right|_{t=0} f \circ \eta_{-t}=-d f(v)=-L_{v} f .
$$

The bracket is thus given by (3.1):

$$
\{F, G\}_{+}(\boldsymbol{M}, \rho)=\int_{\Omega} \boldsymbol{M} \cdot\left[\frac{\delta F}{\delta \boldsymbol{M}}, \frac{\delta G}{\delta \boldsymbol{M}}\right] d x-\int_{\Omega} \rho\left(L_{\delta F / \delta \boldsymbol{M}} \frac{\delta G}{\delta \rho}-L_{\delta G / \delta \boldsymbol{M}} \frac{\delta F}{\delta \rho}\right) d x .
$$

But the left Lie bracket on $\mathfrak{X}$, which shows up in the first term, is minus the standard Lie bracket of vector fields (Abraham and Marsden [1978, example 4.1G]), so this becomes explicitly

$$
\begin{aligned}
\{F, G\}_{+}(\boldsymbol{M}, \rho)= & \int_{\Omega} \boldsymbol{M} \cdot\left(\left(\frac{\delta G}{\delta \boldsymbol{M}} \cdot \nabla\right) \frac{\delta F}{\delta \boldsymbol{M}}-\left(\frac{\delta F}{\delta \boldsymbol{M}} \cdot \nabla\right) \frac{\delta G}{\delta \boldsymbol{M}}\right) d x \\
& +\int_{\Omega} \rho\left(\frac{\delta G}{\delta \boldsymbol{M}} \cdot\left(\nabla \frac{\delta F}{\delta \rho}\right)-\frac{\delta F}{\delta \boldsymbol{M}} \cdot\left(\nabla \frac{\delta G}{\delta \rho}\right)\right) d x
\end{aligned}
$$

This bracket agrees with that of Morrison and Greene [1980]. From the Lie-Poisson equations $\dot{F}=\{F, H\}_{+}$we can obtain the equations of motion directly by choosing $F=\int M_{1} d x, \int M_{2} d x, \int M_{3} d x, \int \rho d x$; the last one represents the equation of continuity and the first three the balance of momentum. We can also get the same result by using (3.2) directly, but with more computations. For example, the term $\rho^{\prime}(\delta H / \delta \mu)^{*} a$ corresponds to $\operatorname{div} \boldsymbol{M}$, as an easy integration by parts argument shows, so that the second component of $X_{H}$ on $\mathfrak{\Im}_{+}^{*}$ yields $\dot{\rho}=-\operatorname{div} \boldsymbol{M}$, which is the continuity equation.

REMARKS. (1) In the same manner, one can treat the case of an incompressible inhomogeneous fluid. The semidirect product in question is now $\mathscr{D}_{\text {vol }} \times \mathscr{F}(\Omega)$, where $\mathcal{D}_{\text {vol }}$ denotes the volume preserving diffeomorphisms of $\Omega$. For the technical details regarding the correct choices of function spaces, see Marsden [1976].

(2) We can also allow $\sigma$, the entropy per unit volume, to be variable. The thermodynamic equation of entropy advection

$$
\frac{\partial \sigma}{\partial t}+\operatorname{div}\left(\frac{\sigma}{\rho} \boldsymbol{M}\right)=0
$$


has to be added to the compressible fluid equations. In addition, the internal energy is $w=w(\rho, \sigma)$ and the pressure is $p=\rho^{2}(\partial w / \partial p+(\sigma / \rho) \partial w / \partial \sigma)$. Thus our system of partial differential equations is (5.3), (5.4), (5.7) with Hamiltonian (5.5) for $w=w(\rho, \sigma)$.

In our framework, this system is Hamiltonian on the dual of the Lie algebra of OD $\times \mathscr{F}(\Omega) \times \mathscr{F}(\Omega)$, where $\mathscr{Q}$ acts on $\mathscr{F}(\Omega) \times \mathscr{F}(\Omega)$ by push-forward in each factor. Hence, the bracket is given by (5.6), to which the term

$$
\int \sigma\left(\left(\frac{\delta G}{\delta M} \cdot \nabla\right) \frac{\delta F}{\delta \sigma}-\left(\frac{\delta F}{\delta M} \cdot \nabla\right) \frac{\delta G}{\delta \sigma}\right) d x
$$

has been added. In this way $\dot{F}=\{F, H\}_{+}$for $F=\int M_{1} d x, \int M_{2} d x, \int M_{3} d x, \int \rho d x$, $\int \sigma d x$ becomes the system (5.3), (5.4), (5.7). One can also see the form of the Lie-Poisson equations directly from (3.2) by remarking that the term $\rho^{\prime}(\delta H / \delta \mu)^{*} a$ corresponds to $(\operatorname{div} \boldsymbol{M}, \operatorname{div}(\sigma \boldsymbol{M} / \rho))$.

We now determine the Clebsch variables for the isentropic flow. It is tempting to use the simplest formula (4.2), but as we shall see below this corresponds to $\boldsymbol{M}=\rho \nabla \phi$, i.e. the flow is required to be potential. Thus we choose the next simplest one given by (4.1). This is a map $J: \widetilde{F}(\Omega) \times \mathscr{F}(\Omega)^{*} \times \mathscr{F}(\Omega) \times \mathscr{F}(\Omega)^{*} \mapsto \mathfrak{S}_{+}^{*}$ given by $J(f, \alpha, g, \beta)=\left(\rho_{f}^{\prime *} \alpha+\rho_{g}^{\prime *} \beta, \beta\right)$. As before, we identify the density $\alpha d x$ with the function $\alpha$, and use $\rho^{\prime}(v) f=-L_{v} f=-\nabla f \cdot v$, so we get $\left\langle\rho_{f}^{\prime *} \alpha, v\right\rangle=-\int \alpha \nabla f \cdot v d x$; i.e. regarded as a vector field, $\rho_{f}^{\prime *} \alpha=-\alpha \nabla f$. Thus

$$
J(f, \alpha, g, \beta)=(-\alpha \nabla f-\beta \nabla g, \beta) .
$$

Now denoting $f=-\mu, g=-\phi, \alpha=\lambda, \beta=\phi$, this Poisson map becomes

$$
(\mu, \lambda, \phi, \rho) \mapsto(\lambda \nabla \mu+\rho \nabla \phi, \rho)=(\boldsymbol{M}, \rho) \tilde{~} .
$$

Hence, we recover the classical Clebsch representation

$$
\boldsymbol{M}=\lambda \nabla \mu+\rho \nabla \phi .
$$

The Poisson bracket of two functions $F, G$ is minus the canonical one because $f=-\mu, g=-\phi$, i.e.

$$
\{F, G\}=\int_{\Omega}\left[\left(\frac{\delta F}{\delta \lambda} \frac{\delta G}{\delta \mu}-\frac{\delta F}{\delta \mu} \frac{\delta G}{\delta \lambda}\right)+\left(\frac{\delta F}{\delta \rho} \frac{\delta G}{\delta \phi}-\frac{\delta F}{\delta \phi} \frac{\delta G}{\delta \rho}\right)\right] d x
$$

In the variables $(\mu, \lambda, \phi, \rho)$, the compressible fluid equations (5.3) and (5.4) take the canonical form

$$
\dot{\lambda}=\frac{\delta \bar{H}}{\delta \mu}, \quad \dot{\mu}=-\frac{\delta \bar{H}}{\delta \lambda}, \quad \dot{\rho}=\frac{\delta \bar{H}}{\delta \phi}, \quad \dot{\phi}=-\frac{\delta \bar{H}}{\delta \rho}
$$

where $\bar{H}(\lambda, \mu, \phi, \rho)=H(\lambda \nabla \mu+\rho \nabla \phi, \rho)$ with $H$ given by (5.5).

If variable entropy is included in the equations, the Clebsch variables are determined by formula (4.3), i.e. we get the classical representation

$$
\boldsymbol{M}=\lambda \nabla \mu+\rho \nabla \phi+\sigma \nabla \psi .
$$

C. Magnetohydrodynamics of an ideal compressible perfectly conducting fluid. We keep the same hypotheses and notation as in Example B but, in addition, we assume that the fluid consists of charged particles in a quasi-neutral state. The configuration 
space remains $\mathscr{D}$, and conservation of mass is unchanged. In the balance of momentum law, one must add the net Lorentz force of the magnetic field created by the fluid in motion. In addition, the hypothesis of infinite conductivity leads one to the conclusion that magnetic lines are frozen in the fluid, i.e. that they are transported along the particle paths. If $\rho$ is the mass density, $v$ the spatial velocity, $\boldsymbol{M}=\rho \boldsymbol{v}$ the momentum density, and $B \in \Lambda^{2}(\Omega)$ the magnetic field regarded as a two-form, then the equations of motion are

$$
\begin{gathered}
M_{i}=-\sum_{j} \frac{\partial}{\partial x_{j}}\left(\frac{M_{i} M_{j}}{\rho}+\delta_{i j}\left(p-\frac{1}{4} \operatorname{Tr} B^{2}\right)-\sum_{k} B_{i k} B_{k j}\right), \\
\dot{\rho}+\operatorname{div} \boldsymbol{M}=0 \text { i.e. } \rho d x+L_{v}(\rho(x) d x)=0 \text { and } \dot{B}+L_{v} B=0
\end{gathered}
$$

where $p=\rho^{2} w^{\prime}(\rho)$ is the pressure and $L_{v}$ the Lie derivative. The last equation just says that $B$ is "frozen" in the fluid. As before, the initial mass density $\rho_{0}$, is given. In addition, the initial magnetic field $B_{0}$ must now be specified. The energy of this fluid is given by

$$
H(\boldsymbol{M}, \rho, B)=\frac{1}{2} \int_{\Omega} \frac{\|M(x)\|^{2}}{\rho(x)} d x+\int_{\Omega} \rho(x) w(\rho)(x) d x+\frac{1}{2} \int_{\Omega}\|B(x)\|^{2} d x
$$

where $\|B\|^{2}=B_{12}^{2}+B_{13}^{2}+B_{23}^{2}$. Since the last two equations of motion are Lie transport equations, the pattern of the previous example shows that the relevant semidirect product is $S=\mathscr{D} \times \mathscr{F}(\Omega) \times \Lambda^{1}(\Omega)$, where $\mathscr{D}$ acts on $\mathscr{F}(\Omega) \times \Lambda^{1}(\Omega)$ by pushing forward on each factor. The Lie algebra is $\mathfrak{I}=\mathfrak{X} \times \mathscr{F}(\Omega) \times \Lambda^{\prime}(\Omega)$, and its dual is $\mathfrak{s}^{*}=\widetilde{S}^{*} \times \mathscr{F}(\Omega)^{*} \times \Lambda^{2}(\Omega)$, the pairing between $\alpha \in \Lambda^{1}(\Omega)$ and $\beta \in \Lambda^{2}(\Omega)$ being $\langle\alpha, \beta\rangle=\int_{\Omega} \alpha \wedge \beta$.

To $H(M, \rho, B)$ there corresponds the Hamiltonian

$$
\begin{aligned}
H_{\rho_{0}, B_{0}}\left(\alpha_{\eta}\right)= & \frac{1}{2}\left\langle\left\langle\alpha_{\eta}, \alpha_{\eta}\right\rangle\right\rangle+\int_{\Omega} \rho_{0}(X) w\left(\rho_{0}(X) J_{\eta}^{-1}(X)\right) d X \\
& +\frac{1}{2} \int_{\Omega}\left\|\left(\eta_{*} B_{0} \circ \eta\right)(X)\right\|^{2} J_{\eta}(X) d X
\end{aligned}
$$

on $T^{*} \mathcal{O} \mathcal{L}$ which is right invariant under the action of

$$
\mathscr{D}_{\rho_{0}, B_{0}}=\left\{\phi \in \mathscr{D} \mid \rho_{0}=\left(\rho_{0} \circ \phi^{-1}\right) J_{\phi^{-1}}, \phi_{*} B_{0}=B_{0}\right\} \text {. }
$$

Thus, Theorem 3.4 for right actions applies, and we conclude that $H(M, \rho, B)$ defines Lie-Poisson equations in $\mathfrak{\mathfrak { s }}_{+}^{*}=\left(\mathfrak{X}^{*} \times \mathscr{F}(\Omega)^{*} \times \Lambda^{2}(\Omega)\right)_{+}$. Recall again that $\mathfrak{X}$ has the left Lie bracket which is minus the usual one. The semidirect product Lie group is $S=\mathscr{D} \times \mathscr{F}(\Omega) \times \Lambda^{\prime}(\Omega)$ where $\mathscr{D}$ acts on $\mathscr{F}(\Omega) \times \Lambda^{\prime}(\Omega)$ by push-forward. As in the previous example, $\mathfrak{X}$ acts on $\mathscr{F}(\Omega) \times \Lambda^{\prime}(\Omega)$ by minus the Lie derivative on each factor. The variables $(\overline{\boldsymbol{M}}, \rho(x) d x, B) \in \mathfrak{S}_{+}^{*}$ are of the following geometric type: $\overline{\boldsymbol{M}}(x)=\rho(x) \boldsymbol{v}^{b}(x) d x \in \mathfrak{X}^{*}$ is a one-form density, $\rho(x) d x \in \mathscr{F}(\Omega)^{*}$ is a density, and $B \in \Lambda^{\prime}(\Omega)^{*}=\Lambda^{2}(\Omega)$ is a two-form.

To write out the Lie-Poisson bracket (3.1) of two functions $F, G: \tilde{\mathfrak{y}}_{+}^{*} \rightarrow \mathbf{R}$, we again identify $\bar{M}(x)$ with $\boldsymbol{M}(x)=\rho(x) v(x), \rho(x) d x$ with $\rho(x)$, and proceed as in 
the previous example. We get

$$
\begin{aligned}
\{F, G\}_{+}(\boldsymbol{M}, \rho, \boldsymbol{B})= & \int_{\Omega} \boldsymbol{M} \cdot\left[\frac{\delta F}{\delta \boldsymbol{M}}, \frac{\delta G}{\delta \boldsymbol{M}}\right] d x-\int_{\Omega} \rho\left(L_{\delta F / \delta \boldsymbol{M}} \frac{\delta G}{\delta \rho}-L_{\delta G / \delta \boldsymbol{M}} \frac{\delta F}{\delta \rho}\right) d x \\
& -\int_{\Omega} B \wedge\left(L_{\delta F / \delta \boldsymbol{M}} \frac{\delta G}{\delta B}-L_{\delta G / \delta \boldsymbol{M}} \frac{\delta F}{\delta B}\right) .
\end{aligned}
$$

The first two terms coincide with (5.6). To bring the third term into a more familiar form, identify $B \in \Lambda^{2}(\Omega)$ with the vector field $\boldsymbol{B}=\left(B_{1}, B_{2}, B_{3}\right)$ by $B_{1}=B_{23}$, $B_{2}=B_{31}, B_{3}=B_{12}$, and identify a one-form $\alpha=\alpha_{1} d x^{1}+\alpha_{2} d x^{2}+\alpha_{3} d x^{3}$ with the corresponding vector field $\left(\alpha_{1}, \alpha_{2}, \alpha_{3}\right)$. A straightforward computation shows that

$$
\begin{aligned}
B \wedge L_{\delta F / \delta M} \frac{\delta G}{\delta B}= & B_{12}\left[\left(\frac{\delta F}{\delta M}\right)_{i} \frac{\delta}{\delta x_{i}}\left(\frac{\delta G}{\delta B}\right)_{3}+\left(\frac{\delta G}{\delta B}\right)_{i} \frac{\partial}{\partial x_{3}}\left(\frac{\delta F}{\delta M}\right)_{i}\right] d x_{1} \wedge d x_{2} \wedge d x_{3} \\
& +B_{31}\left[\left(\frac{\delta F}{\delta M}\right)_{i} \frac{\partial}{\partial x_{i}}\left(\frac{\delta G}{\delta B}\right)_{2}+\left(\frac{\delta G}{\delta B}\right)_{i} \frac{\partial}{\partial x_{2}}\left(\frac{\delta F}{\delta M}\right)_{i}\right] d x_{1} \wedge d x_{2} \wedge d x_{3} \\
& +B_{12}\left[\left(\frac{\delta F}{\delta M}\right)_{i} \frac{\partial}{\partial x_{i}}\left(\frac{\delta G}{\delta B}\right)_{1}+\left(\frac{\delta G}{\delta B}\right)_{i} \frac{\partial}{\partial x_{1}}\left(\frac{\delta F}{\delta M}\right)_{i}\right] d x_{1} \wedge d x_{2} \wedge d x_{3} \\
= & B \cdot\left[\left(\frac{\delta F}{\delta M} \cdot \nabla\right) \frac{\delta G}{\delta B}+\left(\nabla \frac{\delta F}{\delta M}\right) \cdot \frac{\delta G}{\delta B}\right] d x_{1} \wedge d x_{2} \wedge d x_{3}
\end{aligned}
$$

by identifying the one-form $\delta G / \delta B$ with the corresponding vector field with components $(\delta G / \delta B)_{1},(\delta G / \delta B)_{2},(\delta G / \delta B)_{3}$. The Lie-Poisson bracket thus becomes

$$
\begin{aligned}
\{F, G\}_{+}(\boldsymbol{M}, \rho, \boldsymbol{B})= & \int_{\Omega} \boldsymbol{M} \cdot\left[\left(\frac{\delta G}{\delta \boldsymbol{M}} \cdot \nabla\right) \frac{\delta F}{\delta \boldsymbol{M}}-\left(\frac{\delta F}{\delta \boldsymbol{M}} \cdot \nabla\right) \frac{\delta G}{\delta \boldsymbol{M}}\right] d x \\
& +\int_{\Omega} \rho\left[\frac{\delta G}{\delta \boldsymbol{M}} \cdot\left(\nabla \frac{\delta F}{\delta \rho}\right)-\frac{\delta F}{\delta \boldsymbol{M}} \cdot\left(\nabla \frac{\delta G}{\delta \rho}\right)\right] d x \\
& +\int_{\Omega} \boldsymbol{B} \cdot\left[\left(\frac{\delta G}{\delta \boldsymbol{M}} \cdot \nabla\right) \frac{\delta F}{\delta \boldsymbol{B}}-\left(\frac{\delta F}{\delta \boldsymbol{M}} \cdot \nabla\right) \frac{\delta G}{\delta \boldsymbol{B}}\right] d x \\
& +\int_{\Omega} \boldsymbol{B} \cdot\left[\left(\nabla \frac{\delta G}{\delta \boldsymbol{M}}\right) \cdot \frac{\delta F}{\delta \boldsymbol{B}}-\left(\nabla \frac{\delta F}{\delta \boldsymbol{M}}\right) \cdot \frac{\delta G}{\delta \boldsymbol{B}}\right] d x
\end{aligned}
$$

This bracket coincides with the one derived by Morrison and Greene [1980], Holm and Kupershmidt [1982] and Morrison [1982]. With respect to this bracket, the equations are in Lie-Poisson form $\dot{F}=\{F, H\}_{+}$. The equations of motion are obtained by putting $F=\int M_{i} d x, \int \rho d x, \int B_{i} d x, i=1,2,3$.

If entropy is variable, equation (5.7) must be added to the magnetohydrodynamic equations, where $w=w(\rho, \sigma)$ and $p=\rho^{2}(\partial w / \partial \rho+(\sigma / \rho) \partial w / \partial \sigma)$. The Lie-Poisson bracket for this case lives on the dual of the Lie algebra of $\mathscr{D} \times \mathscr{F}(\Omega) \times \Lambda^{1}(\Omega) \times$ $\widetilde{T}(\Omega)$, the action of $\mathscr{Q}$ being push-forward. The bracket has the expression (5.9) to which (5.8) is added.

To obtain the Lie-Poisson description of the magnetohydrodynamic equations when $\operatorname{div} \boldsymbol{B}=0$ and $\boldsymbol{B}=\operatorname{curl} \boldsymbol{A}$, we proceed in the following way. There are two obvious group homorphisms: 


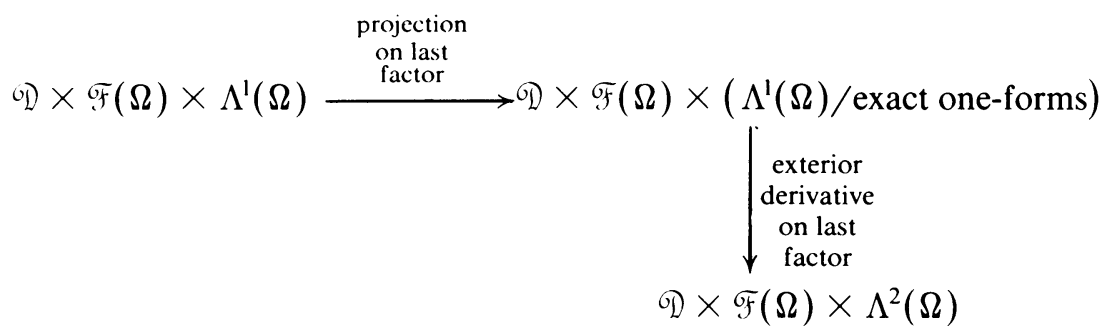

Dualizing the induced Lie algebra mappings, we obtain Poisson maps

$$
\begin{aligned}
\mathfrak{X}^{*} \times \mathscr{F}(\Omega)^{*} \times \Lambda^{2}(\Omega) & \rightarrow \mathfrak{X}^{*} \times \mathscr{F}(\Omega)^{*} \times\left\{\alpha \in \Lambda^{2}(\Omega) \mid d \alpha=0\right\} \\
& \rightarrow \mathfrak{X}^{*} \times \mathscr{F}(\Omega)^{*} \times \Lambda^{1}(\Omega) .
\end{aligned}
$$

In this way we obtain the Lie-Poisson formulation for magnetohydrodynamics in physical variables $(\boldsymbol{M}, \rho, \boldsymbol{B})$, with $\operatorname{div} \boldsymbol{B}=0$ (variables: $\boldsymbol{M}, \rho, \boldsymbol{B})$, or with a magnetic potential (variables: $\boldsymbol{M}, \rho, \boldsymbol{A}$, for $\boldsymbol{B}=\operatorname{curl} \boldsymbol{A}$ ). For the case $\operatorname{div} \boldsymbol{B}=0$ the bracket is still (5.10), whereas for the case $\boldsymbol{B}=\operatorname{curl} \boldsymbol{A}$ the same bracket takes the form

$$
\begin{aligned}
\{F, G\}_{+}(\boldsymbol{M}, \rho, \boldsymbol{A})= & \int_{\Omega} \boldsymbol{M} \cdot\left[\left(\frac{\delta G}{\delta \boldsymbol{M}} \cdot \nabla\right) \frac{\delta F}{\delta \boldsymbol{M}}-\left(\frac{\delta F}{\delta \boldsymbol{M}} \cdot \nabla\right) \frac{\delta G}{\delta \boldsymbol{M}}\right] d x \\
& +\int_{\Omega} \rho\left[\frac{\delta G}{\delta \boldsymbol{M}} \cdot\left(\nabla \frac{\delta F}{\delta \boldsymbol{\rho}}\right)-\frac{\delta F}{\delta \boldsymbol{M}} \cdot\left(\nabla \frac{\delta G}{\delta \boldsymbol{\rho}}\right)\right] d x \\
& +\int_{\Omega}(\nabla \times \boldsymbol{A}) \cdot\left(\frac{\delta F}{\delta \boldsymbol{A}} \times \frac{\delta G}{\delta \boldsymbol{M}}-\frac{\delta G}{\delta \boldsymbol{A}} \times \frac{\delta F}{\delta \boldsymbol{M}}\right) d x \\
& +\int_{\Omega} \boldsymbol{A} \cdot\left[\left(\nabla \cdot \frac{\delta G}{\delta \boldsymbol{A}}\right) \frac{\delta F}{\delta \boldsymbol{M}}-\left(\nabla \cdot \frac{\delta F}{\delta \boldsymbol{A}}\right) \frac{\delta G}{\delta \boldsymbol{M}}\right] d x
\end{aligned}
$$

where we identified the one-form $A$ with the vector field $A=\left(A_{1}, A_{2}, A_{3}\right)$. If variable entropy is present, the term (5.8) is added to the bracket.

To find the Clebsch variables for this problem we use formula (4.2), i.e. $J$ : $\mathscr{F}(\Omega) \times \mathscr{F}(\Omega)^{*} \times \Lambda^{1}(\Omega) \times \Lambda^{2}(\Omega) \rightarrow \mathfrak{F}_{+}^{*}$ is given by

$$
J(f, \alpha, C, B)=\left(\rho_{f, C}^{\prime *}(\alpha, B), \alpha, B\right)=(\boldsymbol{M}, \alpha, B) .
$$

To compute the term $\rho_{f, C}^{\prime *}(\alpha, B)$ we relate $B$ with $B$, and $C$ with $C$. Then integration by parts yields, for $v=\left(v_{1}, v_{2}, v_{3}\right)$,

$$
\begin{aligned}
\rho_{f, \boldsymbol{C}}^{\prime *}(\alpha, \boldsymbol{B}) \cdot \boldsymbol{v} & =\left\langle(\alpha, \boldsymbol{B}), \rho^{\prime}(\boldsymbol{v})(f, C)\right\rangle=-\left\langle(\alpha, \boldsymbol{B}),\left(L_{v} f, L_{v} \boldsymbol{C}\right)\right\rangle \\
& =-\int_{\Omega} \alpha \nabla f \cdot \boldsymbol{v} d x-\int_{\Omega} B \wedge L_{v} C \\
& =-\int_{\Omega} \alpha \nabla f \cdot \boldsymbol{v} d x-\int_{\Omega} \boldsymbol{B} \cdot[(\boldsymbol{v} \cdot \nabla) \boldsymbol{C}+(\nabla \boldsymbol{v}) \cdot \boldsymbol{C}] d x \\
& =-\int_{\Omega} \alpha \nabla f \cdot \boldsymbol{v} d x+\int_{\Omega}[\boldsymbol{C}(\nabla \cdot \boldsymbol{B})+(\boldsymbol{B} \cdot \nabla) \boldsymbol{C}-(\nabla \boldsymbol{C}) \cdot \boldsymbol{B}] \cdot \boldsymbol{v} d x
\end{aligned}
$$


so that $\rho_{f, C}^{\prime *}(\alpha, B)$, regarded as a vector field, equals

$$
\rho_{f, C}^{\prime *}(\alpha, B)=-\nabla f+C(\nabla \cdot B)+(B \cdot \nabla) C-(\nabla C) \cdot B=M .
$$

If we change to the variables $\alpha=\rho, f=-\phi, C=-T$ we obtain the representation

$$
\boldsymbol{M}=\rho \nabla \phi+(\nabla \boldsymbol{T}) \cdot \boldsymbol{B}-\boldsymbol{T}(\nabla \cdot \boldsymbol{B})-(\boldsymbol{B} \cdot \nabla) \boldsymbol{T}
$$

found by Morrison [1982]. The bracket is these variables is minus the canonical one, i.e.

$$
\{\{F, G\}\}(\phi, \rho, \boldsymbol{T}, \boldsymbol{B})=\int_{\Omega}=\left(\frac{\delta F}{\delta \rho} \frac{\delta G}{\delta \phi}-\frac{\delta F}{\delta \phi} \frac{\delta G}{\delta \rho}+\frac{\delta F}{\delta \boldsymbol{B}} \cdot \frac{\delta G}{\delta \boldsymbol{T}}-\frac{\delta F}{\delta \boldsymbol{T}} \cdot \frac{\delta G}{\delta \boldsymbol{B}}\right) d x
$$

With respect to the Hamiltonian

$$
\bar{H}(\phi, \rho, \boldsymbol{T}, \boldsymbol{B})=H(\rho \nabla \phi+(\nabla \boldsymbol{T}) \cdot \boldsymbol{B}-\boldsymbol{T}(\nabla \cdot \boldsymbol{B})-(\boldsymbol{B} \cdot \nabla) \boldsymbol{T}, \rho, \boldsymbol{B}),
$$

the equations of motion for magnetohydrodynamics become the canonical Hamilton equations

$$
\dot{\rho}=\delta \bar{H} / \delta \phi, \quad \dot{\phi}=-\delta \bar{H} / \delta \rho, \quad \dot{\boldsymbol{B}}=\delta \bar{H} / \delta \mathrm{T}, \quad \dot{\boldsymbol{T}}=-\delta \bar{H} / \delta \boldsymbol{B} .
$$

If variable entropy is present, one uses formulas (4.3), which amounts to adding the term $\sigma \nabla \psi$ to the representation of $\boldsymbol{M}$ above.

D. Elasticity. The mathematical framework for the deformation of a body in space is the following (Marsden and Hughes [1983]): $(\mathscr{B}, G)$ and $(\mathcal{S}, g)$ are smooth, oriented, Riemannian manifolds representing the body and space; the configuration space $\mathcal{C}$ consists of the embeddings (not necessarily isometric) of $\mathscr{G}$ in $\delta$. We shall assume here that $\mathscr{B}=\delta=\mathbf{R}^{3}$, deferring to a future paper the more realistic case of a compact body with smooth boundary. In the present situation, $C$ may be identified with the group of diffeomorphisms of $\mathbf{R}^{3}$.

For a given mass density function $\rho_{0}$, the volume element of $\mathscr{B}$ is $\rho_{0}(X) d X$, where $d X$ is the Riemannian volume defined by the metric $G$. The kinetic energy is

$$
\mathscr{K}\left(\alpha_{\phi}\right)=\frac{1}{2} \int_{\varphi B} \rho_{0}(X)\left\|V_{\phi}(X)\right\|^{2} d X
$$

where $\alpha_{\phi}(X)=\rho_{0}(X) V_{\phi}^{b}(X) d X$, and $b: T \mathscr{G}_{B} \rightarrow T^{*} \mathscr{B}$ is the bundle isomorphism induced by $\boldsymbol{G}$. We shall postulate a potential energy of the form

$$
\mathscr{V}(\phi)=\int_{\mathscr{G} B} \phi^{*} W\left(g, \phi_{*} G\right)(X) \rho_{0}(X) d X
$$

where $W$ is the stored energy function and $\phi_{*} \boldsymbol{G}=\boldsymbol{b}$ is called the Finger deformation tensor. (Usually, $W$ is regarded as a function of $G$ and $C=\phi^{*} g$ the Cauchy-Green tensor, but the present description, which is equivalent for isotropic materials, is more convenient for our purposes. A similar formulation in which $W$ depends on $C$ and $\boldsymbol{G}$ can also be given.) With respect to this Hamiltonian, the equations of elastodynamics are Hamiltonian on $T^{*} \mathcal{C}$. From now on we shall deliberately identify $\mathscr{B}=\mathcal{S}=\mathbf{R}^{3}, \mathcal{C}=\mathscr{D}\left(\mathbf{R}^{3}\right)$; for the choice of the correct function spaces, see Hughes, Kato and Marsden [1977].

Let us prove that the potential energy is right invariant under the stabilizer

$$
\mathscr{D}_{\rho_{0}, \boldsymbol{G}}=\left\{\eta \in \mathscr{D}\left(\mathbf{R}^{3}\right) \mid \rho_{0}=\left(\rho_{0} \circ \eta^{-1}\right) J_{\eta^{-1}}, \eta_{*} \boldsymbol{G}=\boldsymbol{G}\right\} .
$$


The change of variables $Y=\eta(X)$ gives $d X=J_{\eta^{-1}}(Y) d Y$ so that

$$
\begin{aligned}
& \tau(\phi \circ \eta)=\int_{\cup \mathfrak{B}} \eta^{*} \phi^{*} W\left(g, \phi_{*} \eta_{*} \boldsymbol{G}\right)(X) \rho_{0}(X) d X \\
& =\int_{G[} \phi^{*} W\left(g, \phi_{*} G\right)(Y)\left(\rho_{0} \circ \eta^{-1}\right)(Y)\left(\rho_{0} \circ \eta^{-1}\right)(Y) J_{\eta^{-1}}(Y) d Y \\
& =\mathscr{V}(\phi) \text {. }
\end{aligned}
$$

In a similar way, it is shown that the kinetic energy is right invariant under this stabilizer.

To apply Theorem 3.4 for right invariant systems, we need to find a left representation of $\mathscr{D}\left(\mathbf{R}^{3}\right)$ on a vector space whose dual should include the densities and the Riemannian metrics on $\mathbf{R}^{3}$. The space of Riemannian metrics is an open cone in the vector space $S_{2}\left(\mathbf{R}^{3}\right)$ of all covariant (indices down) symmetric two-tensors on the body $\mathbf{R}^{3}$. Thus $V$ must be $\mathscr{F}\left(\mathbf{R}^{3}\right) \times S^{2}\left(\mathbf{R}^{3}\right)$, where $S^{2}\left(\mathbf{R}^{3}\right)$ is the vector space of contravariant (indices up) symmetric two-tensors on $\mathbf{R}^{3}$; its dual space is $\mathscr{F}\left(\mathbf{R}^{3}\right)^{*} \times$ $S_{2, d}\left(\mathbf{R}^{3}\right)$, where $S_{2, d}\left(\mathbf{R}^{3}\right)$ is the space of covariant, symmetric two-tensor densities. The pairing between $S^{2}\left(\mathbf{R}^{3}\right)$ and $S_{2, d}\left(\mathbf{R}^{3}\right)$ is given by contraction on both indices, followed by integration over the body $\mathbf{R}^{3}$ with respect to the standard Euclidean measure. The action of $\mathscr{D}\left(\mathbf{R}^{3}\right)$ on $\mathscr{F}\left(\mathbf{R}^{3}\right) \times S^{2}\left(\mathbf{R}^{3}\right)$ is push-forward; thus, the induced action on the dual will also be push-forward. Theorem 3.4 can now be applied since the Hamiltonian $\mathscr{K}\left(\alpha_{\phi}\right)+\mathcal{V}(\phi)$ depends smoothly on $\rho_{0}$ and $\boldsymbol{G}$. Thus, we get the equations of elastodynamics in $\mathbf{R}^{3}$ as a Lie-Poisson system on $\mathfrak{S}_{+}^{*}$, where $\mathfrak{g}$ is the Lie algebra of the semidirect product group $\mathcal{S}=\mathscr{D}\left(\mathbf{R}^{3}\right) \times \mathscr{F}\left(\mathbf{R}^{3}\right) \times S^{2}\left(\mathbf{R}^{3}\right)$. The density $\rho$ and deformation strain $\boldsymbol{b}$ are obtained from the initial density $\rho_{0}$ and metric $\boldsymbol{G}$ by push-forward, i.e. $\rho(x) d x=\phi_{*}\left(\rho_{0}(X) d X\right)$, and $\boldsymbol{b}=\phi_{*} \boldsymbol{G}$. The corresponding element of $S_{2, d}$ that is used is $\boldsymbol{\beta}=\boldsymbol{b} \otimes \rho d x$.

The bracket on $\mathfrak{S}_{+}^{*}$ for $F, G: \mathfrak{\Im}^{*} \rightarrow \mathbf{R}$ is given by (3.1), i.e.

$$
\begin{aligned}
\{F, G\}_{+}(\boldsymbol{M}, \rho, \boldsymbol{\beta})= & \int_{\phi} \boldsymbol{M} \cdot\left[\left(\frac{\delta G}{\delta \boldsymbol{M}} \cdot \nabla\right) \frac{\delta F}{\delta \boldsymbol{M}}-\left(\frac{\delta F}{\delta \boldsymbol{M}} \cdot \nabla\right) \frac{\delta G}{\delta \boldsymbol{M}}\right] d x \\
& +\int_{\phi} \rho\left[\frac{\delta G}{\delta \boldsymbol{M}} \cdot\left(\nabla \frac{\delta F}{\delta \rho}\right)-\frac{\delta F}{\delta \boldsymbol{M}} \cdot\left(\nabla \frac{\delta F}{\delta \rho}\right)\right] d x \\
& -\int_{\phi} \rho\left(L_{\delta F / \delta \boldsymbol{M}} \frac{\delta G}{\delta \boldsymbol{\beta}}-L_{\delta G / \delta \boldsymbol{M}} \frac{\delta F}{\delta \boldsymbol{\beta}}\right) d x
\end{aligned}
$$

note that here $\delta F / \delta \boldsymbol{\beta}$ and $\delta G / \delta \boldsymbol{\beta}$ belong to $S^{2}\left(\mathbf{R}^{3}\right)$, the dual space of $S_{2, d}\left(\mathbf{R}^{3}\right)$.

As in the previous examples, one can consider, as well, the case of variable entropy. Clebsch representation can be found for this problem by formula (4.1); the momentum density $M$ will have a quite long expression involving indices, since $b$ has six components. A related construction for elasticity, starting with Clebsch variables and using different physical variables, was given by Holm and Kupershmidt [1982]. As they pointed out, the mapping $(\boldsymbol{M}, \rho, \boldsymbol{\beta}=\boldsymbol{b} \otimes \rho d x) \mapsto(\boldsymbol{M}, \rho, \boldsymbol{C}$ $\otimes \rho d x$ ) where $C$ is the Cauchy-Green tensor (their $e$ ) defines a canonical map between our bracket and theirs (formula (88)). 
6. Semidirect products in electromagnetic coupling. In Marsden and Weinstein [1982a], two realizations of the reduced space for the Maxwell-Vlasov equation were given. The Poisson brackets for these two realizations are reproduced here:

$$
\begin{aligned}
\{F, G\}(f, \boldsymbol{E}, \boldsymbol{B})= & \int f\left\{\frac{\delta F}{\delta f}, \frac{\delta G}{\delta f}\right\} d \boldsymbol{x} d \boldsymbol{v}+\int\left(\frac{\delta F}{\delta \boldsymbol{E}} \operatorname{curl} \frac{\delta G}{\delta \boldsymbol{B}}-\frac{\delta G}{\delta \boldsymbol{E}} \operatorname{curl} \frac{\delta F}{\delta \boldsymbol{B}}\right) d \boldsymbol{x} \\
& +\int\left(\frac{\delta F}{\delta \boldsymbol{E}} \cdot \frac{\partial f}{\partial \boldsymbol{v}} \frac{\delta G}{\delta f}-\frac{\delta G}{\delta \boldsymbol{E}} \cdot \frac{\partial f}{\partial \boldsymbol{v}} \frac{\delta F}{\delta f}\right) d \boldsymbol{x} d \boldsymbol{v} \\
+ & \int f \boldsymbol{B} \cdot\left(\frac{\partial}{\partial \boldsymbol{v}} \frac{\delta F}{\delta f} \times \frac{\partial}{\partial \boldsymbol{v}} \frac{\delta G}{\delta f}\right) d \boldsymbol{x} d \boldsymbol{v} . \\
& +\int\left(\frac{\delta F}{\delta \boldsymbol{E}} \operatorname{curl} \frac{\delta G}{\delta \boldsymbol{B}}-\frac{\delta G}{\delta \boldsymbol{E}} \operatorname{curl} \frac{\delta F}{\delta \boldsymbol{B}}\right) d \boldsymbol{x} \\
& +\left[\int f_{\mathrm{mom}}\left(\frac{\partial}{\partial \boldsymbol{p}} \frac{\delta F}{\delta f_{\mathrm{mom}}}\right)\left(\frac{\partial}{\partial \boldsymbol{x}} \Delta^{-1} \operatorname{div} \frac{\delta G}{\delta \boldsymbol{E}}\right) d \boldsymbol{x} d \boldsymbol{p}\right. \\
& \left.\left.\left.\left.-\int f_{\mathrm{mom}}, \boldsymbol{E}, \boldsymbol{B}\right)=\frac{\delta G}{\delta f_{\mathrm{mom}}}\right\} d \boldsymbol{x} d \frac{\partial}{\partial \boldsymbol{p}} \frac{\delta F}{\delta f_{\mathrm{mom}}}\right) \frac{\partial}{\partial \boldsymbol{x}}\left(\Delta^{-1} \operatorname{div} \frac{\delta F}{\delta \boldsymbol{E}}\right) d \boldsymbol{x} d \boldsymbol{p}\right] .
\end{aligned}
$$

The bracket (6.1) arises from a direct and natural identification of the reduced space with the space of plasma distribution function $f(\boldsymbol{x}, \boldsymbol{v}, t)$ in position-velocity space, and electric and magnetic fields. In the second case a gauge condition $\operatorname{div} \boldsymbol{A}=0$ is chosen and the distribution function is regarded as a function of position and momentum $f_{\text {mom }}(\boldsymbol{x}, \boldsymbol{p}, t)$.

One can see directly that the first, third and fourth terms of (6.2) correspond to the Lie-Poisson structure for the semidirect product $\mathfrak{A} \times \mathfrak{g}$ where $\mathfrak{A}$ is the abelian Lie algebra of vector potentials $\boldsymbol{A}$ and $\mathfrak{S}$ is the Lie algebra of functions under the Poisson bracket. The action of $\mathfrak{A}$ on $\mathfrak{S}$ is by $(\boldsymbol{A}, f) \mapsto\{\phi, f\}$, where $\phi=\Delta^{-1} \operatorname{div} \boldsymbol{A}$. One reason this second representation (6.2) is useful is because, unlike $f, f_{\text {mom }}$ evolves in $\mathfrak{S}^{*}$ along a coadjoint orbit; i.e. by composition with canonical transformations.

In this section we give an abstract construction which includes the above observations about the bracket (6.2) as a special case.

The bracket (6.2) can be constructed using ideas from Sternberg [1977]. He constructs a certain symplectic quotient space using a connection on a principal bundle. In Weinstein [1978] a reduced space independent of any choice of connection is constructed, which is symplectically diffeomorphic to Sternberg's space. (See Marsden [1981, pp. 35-37] for an account.) In fact, (6.2) is essentially Sternberg's version of the bracket with the connection given by the choice of gauge. The main result of this section says that for reductions by abelian groups, Sternberg's bracket is a semidirect product bracket plus a canonical bracket. We shall give a new application to multifluid electrodynamics below. We emphasize that, apparently, this 
semidirect product structure arises for quite different reasons from those considered earlier in this paper.

We use the following notation for the abstract result. Let $\mathfrak{A}$ and $\mathcal{G}$ be vector spaces and

$$
D: \mathcal{G} \rightarrow \mathfrak{U} \quad \text { and } P: \mathfrak{A} \rightarrow \mathcal{G}
$$

be given linear maps with $P \circ D=$ id. (For $\mathfrak{A}$ the space of vector potentials, $\mathcal{G}$ is the space of gauge transformations $\phi$ and $D(\phi)=d \phi$, while $P(A)=\Delta^{-1} \delta A$ is the map associated to Hodge projection.) Let $\mathfrak{g}$ be a Lie algebra and $R: \mathcal{G} \rightarrow \operatorname{Aut}(\mathfrak{g})$ a representation of $\mathcal{G}$ as an abelian group on $\mathfrak{S}$.

Now $\mathcal{G}$ acts on $\mathfrak{A}$ by $\phi \cdot A=A+D \phi$ and the induced action on $T^{*} \mathfrak{U}=\mathfrak{U} \times \mathfrak{U} *$ is $\phi \cdot(A, Y)=(A+D \phi, Y)$ with momentum map $(A, Y) \mapsto D^{*} Y$. For $\rho \in \mathcal{G}^{*}$, the reduced manifold is

$$
\left(T^{*} \mathfrak{A}\right)_{\rho} \cong \mathfrak{A} / D(\mathcal{G}) \times\left(D^{*}\right)^{-1}(\rho),
$$

the identification being induced by the map $(A, Y) \mapsto([A],-Y)=(B, E)$ where $[A]=B$ is the equivalence class of $A$. As in the case of Maxwell's equations treated in Marsden and Weinstein [1982a], the bracket on the reduced space (6.3) is readily computed to be

$$
\{F, G\}(B, E)=\left\langle\left[\frac{\delta F}{\delta E}\right], \frac{\delta G}{\delta B}\right\rangle-\left\langle\left[\frac{\delta G}{\delta E}\right], \frac{\delta F}{\delta B}\right\rangle .
$$

Consider the manifold $T^{*} \mathfrak{A} \times \mathfrak{s}^{*}$ with its bracket

$$
\{F, G\}(A, Y, \mu)=\left\langle\frac{\delta F}{\delta A}, \frac{\delta G}{\delta Y}\right\rangle-\left\langle\frac{\delta G}{\delta A}, \frac{\delta F}{\delta Y}\right\rangle \pm\left\langle\mu,\left[\frac{\delta F}{\delta \mu}, \frac{\delta G}{\delta \mu}\right]\right\rangle .
$$

We are interested in reducing $T^{*} \mathfrak{A} \times \mathfrak{s}_{ \pm}^{*}$ by the induced $\mathcal{S}$ action; the action on $T^{*} \mathfrak{U}$ is as above and the action on $\mathfrak{s}^{*}$ is $\phi \cdot \mu=R_{*}(\phi) \cdot \mu=\mu \circ R(-\phi)$. The induced momentum map is

$$
J: T^{*} \mathfrak{A} \times \mathfrak{\Im}_{ \pm}^{*} \rightarrow \mathcal{G}^{*}, \quad J(A, Y, \mu)=D^{*} Y+J_{\mathfrak{5}}(\mu),
$$

where $J_{\mathfrak{s}}$ is an equivariant momentum map for the action of $\mathcal{G}$ on $\mathfrak{s}_{+}^{*}$.

6.1. Theorem. Let $\rho \in \mathcal{G}^{*}$. The reduced manifold $\left(T^{*} \mathfrak{U} \times \mathfrak{S}_{ \pm}^{*}\right)_{\rho}$ is Poisson diffeomorphic to

$$
\mathcal{P}=\left\{(B, E, \mu) \in \mathfrak{A} / \mathcal{G} \times \mathfrak{A}^{*} \times \mathfrak{5}_{ \pm}^{*} \mid D^{*} E=J_{\mathfrak{s}}(\mu)-\rho\right\}
$$

where $\mathcal{P}$ has the following Poisson structure:

$$
\begin{aligned}
\{F, G\}(B, E, \mu)= & \left\langle\left[\frac{\delta F}{\delta E}\right], \frac{\delta G}{\delta B}\right\rangle-\left\langle\left[\frac{\delta G}{\delta E}\right], \frac{\delta F}{\delta B}\right\rangle \\
& \pm\left\langle\mu,\left[\frac{\delta F}{\delta \mu}, \frac{\delta G}{\delta \mu}\right]\right\rangle+\left\langle\mu,\left(R^{\prime} \circ P\right)\left(\frac{\delta F}{\delta E}\right) \cdot \frac{\delta G}{\delta \mu}\right\rangle \\
& -\left\langle\mu,\left(R^{\prime} \circ P\right)\left(\frac{\delta G}{\delta E}\right) \cdot \frac{\delta F}{\delta \mu}\right\rangle .
\end{aligned}
$$

The first two terms of (6.8) coincide with (6.4), the reduced bracket on $\left(T^{*} \mathfrak{A}\right)_{\rho}$, while the last three terms coincide with the Lie-Poisson bracket on the dual of the semidirect product Lie algebra $\mathfrak{A} \times \mathfrak{s}$ with $\mathfrak{A}$ acting on $\mathfrak{s}$ by $R^{\prime} \circ P$. 
6.2. RemarKs. This result can be generalized to the following setting, appropriate for Yang-Mills fields: $\mathcal{G}$ is a Lie group with Lie algebra $\mathfrak{g}, \mathfrak{A}$ is an affine space based on the underlying vector space $\mathfrak{A}$, and $\mathcal{G}$ acts on $\mathfrak{A}$. This action is assumed to be by affine transformations, so embeds $\mathfrak{a} \rightarrow \mathfrak{A}$ by a map $D: \mathfrak{g} \rightarrow \mathfrak{A}$. Let $P: \mathfrak{H} \rightarrow \mathfrak{g}$ be a projection map and $\rho \in \mathrm{q}^{*}$. Then

$$
\stackrel{\rho}{\rho}=\left\{(B, E, \mu) \in \mathfrak{H} / \mathcal{G} \times \mathfrak{U}^{*} \times \mathfrak{S}^{*} \mid D^{*} E=J_{s}(\mu)-\rho\right\}
$$

and the formula (6.8) still holds. Here $R: \mathcal{G} \rightarrow \operatorname{Aut}(\mathfrak{g})$ is a (nonabelian) group representation and $R^{\prime}: \mathfrak{g} \rightarrow \operatorname{Der}(\mathfrak{s})$ is the induced Lie algebra representation.

Proof OF 6.1. Define the map $\Phi: J^{-1}(\rho) \subset T^{*} \mathfrak{A} \times \mathfrak{S}_{ \pm}^{*} \rightarrow \mathscr{P}$ by

$$
\Phi(A, Y, \mu)=\left(B, E, R(P(A))^{*} \mu\right)
$$

where $B=[A], E=-Y$. Note that the image lies in op since

$$
D^{*} E-J_{\check{\xi}}\left(R(P(A))^{*} \mu\right)=-D^{*} Y-J_{\tilde{\xi}}(\mu)=-J(A, Y, \mu)=-\rho
$$

by equivariance of $J_{\xi}$. (The map $\Phi$ is closely related to the map between the Weinstein and Sternberg spaces discussed earlier.) Now $\Phi$ is $\mathcal{G}$ invariant:

$$
\begin{aligned}
\Phi\left(A+D \phi, Y, R(\phi)_{*} \mu\right) & =\left([A+D \phi], E, R(P(A+D \phi))^{*} R(\phi)_{*} \mu\right) \\
& =\left([A], E, R(P(A)+\phi)^{*} R(\phi)_{*} \mu\right) \\
& =\left([A], E, R(P(A))^{*} \mu\right)=\Phi(A, Y, \mu) .
\end{aligned}
$$

It is readily checked that the induced map from $J^{-1}(\rho) / \mathcal{G}$ to $\mathscr{\rho}$ is a diffeomorphism. To compute the Poisson structure on $\mathscr{P}$, let $F, G: \mathcal{P} \rightarrow \mathbf{R}$ and let $\bar{F}, \bar{G}$ : $J^{-1}(\rho) \rightarrow \mathbf{R}$ be given by $\bar{F}=F \circ \Phi, \bar{G}=G \circ \Phi$, extended off $J^{-1}(\rho)$ in an arbitrary way. By a straightforward computation using the chain rule, one finds that at $(A, Y, \mu) \in J^{-1}(\rho)$,

$$
\begin{aligned}
\left\langle\frac{\delta \bar{F}}{\delta A}, A^{\prime}\right\rangle & =\left\langle\frac{\delta F}{\delta B},\left[A^{\prime}\right]\right\rangle+\left\langle\nu \cdot\left(R^{\prime} \circ P\right)\left(A^{\prime}\right) \cdot \frac{\delta F}{\delta \nu}\right\rangle, \\
\frac{\delta \bar{F}}{\delta Y} & =-\frac{\delta F}{\delta E}, \quad \frac{\delta \bar{F}}{\delta \mu}=R(P(A)) \cdot \frac{\delta F}{\delta \nu},
\end{aligned}
$$

where $B=[A], E=-Y$ and $\nu=R(P(A))^{*} \mu$. Substitution of (6.9) into the bracket (6.5) for $\{\bar{F}, \bar{G}\}$ yields $(6.8)$.

6.3. ExAmples. A. The Maxwell-Vlasov bracket (6.2) is a special case of (6.8) by choosing $\mathfrak{A}$ to be the one-forms (vector potentials) $\boldsymbol{A}$ on $\mathbf{R}^{3}, \mathcal{G}$ the functions $\phi$ on $\mathbf{R}^{3}$ and $\mathfrak{S}$ the functions $f$ on $\mathbf{R}^{6}$ with bracket $\{f, g\}$. The map $D$ is $\phi \mapsto d \phi, P(\boldsymbol{A})=$ $-\Delta^{-1}(\operatorname{div} \boldsymbol{A})$ and $(R(\phi) \cdot f)(\boldsymbol{x}, \boldsymbol{p})=f(\boldsymbol{x}, \boldsymbol{p}+d \phi)$. Clearly $R^{\prime}(\phi) \cdot f=\{\phi, f\}$. Thus (6.8) reduces to $(6.2)$.

In this case, observe that each of the last two terms of (6.2) have the form of a tangent vector to a coadjoint orbit on $\mathfrak{Z}_{+}^{*}$ unlike (6.1). Thus in (6.2), $f_{\text {mom }}$ evolves in such a way that it remains on coadjoint orbits in $\mathfrak{S}_{+}^{*}$.

B. Next we consider the case of the Euler-Maxwell equations (multispecies fluid electrodynamics). We reproduce the bracket here from Spencer [1982] and Marsden, 
Weinstein, Ratiu, Schmid and Spencer [1983], with the entropy and species label suppressed only for notational simplicity:

$$
\begin{aligned}
\{F, G\}(\boldsymbol{M}, \rho, \boldsymbol{E}, \boldsymbol{B})= & \int \boldsymbol{M} \cdot\left[\left(\frac{\delta G}{\delta M} \cdot \nabla\right) \frac{\delta F}{\delta M}-\left(\frac{\delta F}{\delta M} \cdot \nabla\right) \frac{\delta G}{\delta M}\right] d x \\
& +\int \rho\left[\frac{\delta G}{\delta M} \cdot\left(\nabla \frac{\delta F}{\delta \rho}\right)-\frac{\delta F}{\delta M} \cdot\left(\nabla \frac{\delta G}{\delta \rho}\right)\right] d x \\
& +\int\left(\frac{\delta F}{\delta E} \cdot \operatorname{curl} \frac{\delta G}{\delta B}-\frac{\delta G}{\delta E} \cdot \operatorname{curl} \frac{\delta F}{\delta B}\right) d x \\
= & \int\left[\frac{\delta F}{\delta M} \cdot \frac{\delta G}{\delta E}-\frac{\delta G}{\delta M} \cdot \frac{\delta F}{\delta E}+B \cdot\left(\frac{\delta F}{\delta M} \times \frac{\delta G}{\delta M}\right) a \rho\right] d x,
\end{aligned}
$$

where $a$ is the charge to mass ratio of the particles. This bracket arises by reducing the canonical bracket on $(\mathfrak{X} \times \mathscr{F})_{+}^{*} \times T^{*} \mathfrak{A}$ (compressible flow plus electromagnetic potentials) relative to the gauge group of electromagnetism. The unreduced variables are $(\boldsymbol{N}, \rho, \boldsymbol{A}, \boldsymbol{Y})$ where $\boldsymbol{N}$ is the fluid momentum density, related to the velocity density $\boldsymbol{M}$ by $\boldsymbol{M}=\boldsymbol{N}-a \rho \boldsymbol{A}$. In the derivation of (6.10), $\boldsymbol{N}$ gets mapped to $\boldsymbol{M}$ by the reduction procedure. However, if we use the bracket (6.8), the identification of the reduced space is different and $N$ remains. Here, $\mathfrak{A}, \mathcal{G}, P$ and $D$ are as above, while $\mathfrak{s}=\mathfrak{X} \times \mathscr{F}$, carrying the bracket (5.6) with $\boldsymbol{M}$ replaced by $\boldsymbol{N}$. The representation $R$ is given by

$$
R(\phi) \cdot(N, \rho)=(N+a \rho d \phi, \rho) .
$$

Observing that $R^{\prime}(\phi) \cdot(N, \rho)=(a \rho d \phi, 0)$, we find that (6.8) becomes

$$
\begin{aligned}
\{F, G\}(B, E, N, \rho)= & \int\left(\frac{\delta F}{\delta E} \operatorname{curl} \frac{\delta G}{\delta B}-\frac{\delta G}{\delta E} \operatorname{curl} \frac{\delta F}{\delta B}\right) d x \\
& +\int N \cdot\left[\frac{\delta G}{\delta N} \cdot\left(\nabla \frac{\delta F}{\delta N}\right)-\frac{\delta F}{\delta N} \cdot \nabla \frac{\delta G}{\delta N}\right] d x \\
& +\int \rho\left[\frac{\delta G}{\delta N} \cdot\left(\nabla \frac{\delta F}{\delta \rho}\right)-\frac{\delta F}{\delta N} \cdot\left(\nabla \frac{\delta G}{\delta \rho}\right)\right] d x \\
& +\int N \cdot\left[a \frac{\delta F}{\delta \rho} d \Delta^{-1} \operatorname{div} \frac{\delta G}{\delta E}-a \frac{\delta G}{\delta \rho} d \Delta^{-1} \operatorname{div} \frac{\delta F}{\delta E}\right] d x
\end{aligned}
$$

In this momentum representation, the last two terms of (6.11) represent the fluid-electromagnetic coupling. They are different from $(6.10)$ in that now the $(N, \rho)$ equations are modified merely by adding a Lie derivative to the $\dot{\rho}$ equation. Thus, $(N, \rho)$ evolve in such a way that they remain on coadjoint orbits in $(\mathfrak{X} \times \mathscr{F})_{+}^{*}$. This is consistent with the corresponding result for plasmas in view of the fact that $\left(f_{\text {mom }}, \boldsymbol{E}, \boldsymbol{B}\right)$ and $(\boldsymbol{N}, \rho, \boldsymbol{E}, \boldsymbol{B})$ are related by a map induced from a naturally constructed momentum map (Marsden, Weinstein, Ratiu, Schmid and Spencer [1983, Theorem 10.3]).

ACKNOWLEDGEMENTs. We would like to thank Darryl Holm, Boris Kupershmidt and Phil Morrison for useful discussions and correspondence. 


\section{REFERENCES}

R. Abraham and J. E. Marsden [1978], Foundations of mechanics, 2nd ed., Addison-Wesley, Reading, Mass.

F. P. Bretherton [1970], A note on Hamilton's principle for perfect fluids, J. Fluid Mech. 44, 19-31.

I. E. Dzyaloshinskii and G. E. Volovick [1980], Poisson brackets in condensed matter physics, Ann. Physics 125, 67-97.

D. G. Ebin and J. Marsden [1970], Groups of diffeomorphisms and the motion of an incompressible fluid. Ann. of Math. (2) 92, 102-163.

V. Guillemin and S. Sternberg [1980]. The moment map and collective motion, Ann. Physics 127, $220-253$

[1982] Symplectic techniques in physics (book in preparation).

D. Holm and B. Kupershmidt [1983], Poisson brackets and Clebsch representations for magnetohydrodynamics, multifluid plasmas and elasticity, Physica D (to appear).

P. J. Holmes and J. E. Marsden [1983], Horseshoes and Arnold diffusion for Hamiltonian systems on Lie groups, Indiana Univ. Math. J. 32, 273-310.

T. Hughes, T. Kato and J. Marsden [1977], Well-posed quasilinear second-order hyperbolic systems with applications to nonlinear elastodynamics and general relativity, Arch. Rational Mech. Anal. 63, 273-294.

D. Kazhdan, B. Kostant and S. Sternberg [1978], Hamiltonian group actions and dynamical sy'stems of Calogero type, Comm. Pure Appl. Math. 31, 481-508.

B. Kupershmidt [1982], Discrete Lax equations and differential-difference equations (lecture notes).

S. Lie [1890], Theorie der Transformationsgruppen, Zweiter Abschnitt, Teubner, Leipzig.

J. E. Marsden [1976], Well-posedness of the equations of a non-homogeneous perfect fluid, Comm. Partial Differential Equations 1, 215-230.

[1981], Lectures on geometric methods in mathematical physics, CBMS-NSF Regional Conf. Ser.

in Appl. Math., No. 37, SIAM, Philadelphia, Pa.

J. E. Marsden and T. J. R. Hughes [1983], Mathematical foundations of elasticity, Prentice-Hall, Englewood Cliffs, N.J.

J. E. Marsden and A. Weinstein [1974], Reduction of symplectic manifolds with symmetry, Rep. Math. Phys. 5, 121-130.

[1982a], The Hamiltonian structure of the Maxwell-Vlasov equations, Physica D 4, 394-406.

[1982b], Coadjoint orbits, vortices and Clebsch variables for incompressible fluids, Physica D (to appear).

J. E. Marsden, A. Weinstein, T. Ratiu, R. Schmid and R. G. Spencer [1983], Hamiltonian sistems with symmetry, coadjoint orbits and plasma physics, Proc. IUTAM-ISIMM Sympos. (Modern Developments in Analytical Mechanics, Torino, June 7-11, 1982) (to appear).

P. J. Morrison [1982], Poisson brackets for fluids and plasmas, Mathematical Methods in Hydrodynamics and Integrability in Related Dynamical Systems (M. Tabor and Y. M. Treve, eds.), AIP Conf. Proc. (La Jolla Institute, 1981) 88, 13-46.

P. J. Morrison and J. M. Greene [1980], Noncanonical hamiltonian density formulation of hydrodinamics and ideal magnetohydrodinamics, Phys. Rev. Letters, 45, 790-794.

Г. Ratiu [1980], Euler-Poisson equations on Lie algebras. Thesis, Univ. of Calif., Berkeley.

[1981], Euler-Poisson equations on Lie algebras and the $N$-dimensional heavy rigid bodl. Proc. Nat. Acad. Sci. U.S.A. 78, 1327-1328.

[1982], Euler-Poisson equations on Lie algebras and the $N$-dimensional heavy rigid body. Amer. J.

Math. 104, 409-448.

J. H. Rawnsley [1975], Representations of a semi-direct product by quantization, Math. Proc. Cambridge. Philos. Soc. 75, 345-350.

R. L. Seliger and G. B. Whitham [1968], Variational principles in continuum mechanics, Proc. Roy. Soc. London Ser. A 305, 1-25.

R. G. Spencer [1982], The Hamiltonian structure of multi-species fluid electrodynamics, Mathematical Methods in Hydrodynamics and Integrability in Related Dynamical Systems (M. Tabor and V. M. Treve, eds.), AIP Conf. Proc. (La Jolla Institute, 1981) 88, 121-126.

S. Sternberg [1977], On minimal coupling and the symplectic mechanics of a classical particle in the presence of a Yang-Mills field, Proc. Nat. Acad. Sci. 74, 5253-5254.

A. M. Vinogradov and B. Kupershmidt [1977], The structure of Hamiltonian mechanics, Russian Math. Surveys 32, 177-243. 
A. Weinstein [1978], A universal phase space for particles in Yang-Mills fields, Lett. Math. Phys. 2 , 417-420.

[1982], The local structure of Poisson manifolds, J. Differential Geometry (to appear).

Department of Mathematics, University of California, Berkeley, California 94720 (Current address of J. E. Marsden and Alan Weinstein)

Department of Mathematics, University of Michigan, Ann Arbor, Michigan 48109

Current address (Tudor Ratiu): Department of Mathematics, University of Arizona, Tucson, Arizona 85721 